\title{
Chaotic strings and standard model parameters
}

\author{
Christian Beck| \\ Isaac Newton Institute for Mathematical Sciences \\ University of Cambridge \\ 20 Clarkson Road \\ Cambridge, CB3 0EH, UK
}

\begin{abstract}
We introduce so-called chaotic strings (coupled 1-dimensional noise strings underlying the Parisi-Wu approach of stochastic quantization on a small scale) as a possible amendment of ordinary string theories. These strings are strongly self-interacting and exhibit strongest possible chaotic behavior. Constraints on the vacuum energy of the strings fix a certain discrete set of allowed string couplings. We provide extensive numerical evidence that these string couplings numerically coincide with running standard model coupling constants, evaluated at energy scales given by the masses of the known quarks, leptons and gauge bosons. Chaotic strings can thus be used to provide a theoretical argument why certain standard model parameters are realized in nature, others are not, assuming that the a priori free standard model couplings evolve to the minima of the effective potentials. The chaotic string spectrum correctly reproduces the numerical values of the electroweak and strong coupling constants with a precision of 4-5 digits, as well as the (free) masses of the known quarks and leptons with a precision of 3-4 digits. Neutrino mass predictions consistent with present experiments are obtained. The $W$ boson mass also comes out correctly, and a Higgs mass prediction is obtained.
\end{abstract}

\footnotetext{
${ }^{1}$ Permanent address: School of Mathematical Sciences, Queen Mary, University of London, Mile End Road, London E1 4NS, UK
} 


\section{Introduction}

A fundamental problem of particle physics is the fact that there are about 25 free fundamental constants which are not understood on a theoretical basis. These constants are essentially the values of the three coupling constants, the quark and lepton masses, the $W$ and Higgs boson mass, and various mass mixing angles. An explanation of the observed numerical values is ultimately expected to come from a larger theory that embeds the standard model. Prime candidates for this are superstring and $M$ theory [1]-[3]. However, so far the predictive power of these and other theories is not large enough to allow for precise numerical predictions.

In this paper we will report on a numerical observation that may shed more light on this problem. We have found that there is a simple class of $1+1$ dimensional strongly self-interacting discrete field theories (called 'chaotic strings' in the following) that have a remarkable property. The expectation of the vacuum energy of these strings is minimized for string couplings that numerically coincide with running standard model couplings $\alpha(E)$, the energy $E$ being given by the masses of the known quarks, leptons, and gauge bosons. Chaotic strings can thus be used to provide theoretical arguments why certain standard model parameters are realized in nature, others are not. We may assume that the a priori free parameters evolve to the local minima of the effective potentials generated by the chaotic strings. Out of the many possible vacua, chaotic strings may select the physically relevant vacuum of superstring theories.

The dynamics of the chaotic strings is discrete in both space and time and exhibits strongest possible chaotic behaviour. It can be regarded as a dynamics of vacuum fluctuations that can be used to 2nd-quantize other fields, for example ordinary standard model fields, or ordinary strings, by dynamically generating the noise of the Parisi-Wu approch of stochastic quantization «4 5] on a very small scale. Mathematically, chaotic strings are coupled map lattices [6] of diffusively coupled Tchebyscheff maps $T_{N}$ of order N. It turns out that there are six different relevant chaotic string theories - similar to the six components that make up M-theory in the moduli space of superstring theory [3]. We will label these six chaotic string theories as $3 A, 3 B, 2 A, 2 B, 2 A^{-}, 2 B^{-}$. Here the first number denotes the index $N$ of the Tchebyscheff polynomial and the letter A,B distinguishes between the

forward and backward coupling form. The index - denotes anti-diffusive coupling. Though in principle one can study these string theories for arbi- 
trary $N$, for stochastic quantization only the cases $N=2$ and $N=3$ yield non-trivial behaviour in a first and second order perturbative approach [7, 8].

Chaotic strings can be used to generate effective potentials for self-interacting scalar (dilaton-like) fields. They may be effectively used to break supersymmetry via second quantization effects (though a concrete embedding into superstring or $M$ theory is still a long theoretical way to go, if any such embedding exists at all). Assuming that the a priori free standard model couplings evolve to the minima of the effective potentials generated by the chaotic strings, one can obtain a large number of very precise predictions. The smallest stable zeros of the expectation of the interaction energy of the chaotic $3 A$ and $3 B$ strings are numerically observed to coincide with the running electroweak couplings at the smallest fermionic mass scales. Inverting the argument, the chaotic $3 \mathrm{~A}$ string can be used to theoretically predict that the low-energy limit of the fine structure constant has the numerical value $\alpha_{e l}(0)=0.0072979(17)=1 / 137.03(3)$, to be compared with the experimental value $1 / 137.036$. The $3 B$ string predicts that the effective electroweak mixing angle is numerically given by $\bar{s}_{l}^{2}=\sin ^{2} \theta_{\text {eff }}^{\text {lept }}=0.23177(7)$, in perfect agreement with the experimental measurements at LEP, which yield the value $\bar{s}_{l}^{2}=0.23185(23)$ [9]. The smallest stable zeros of the interaction energy of the $N=2$ strings are observed to coincide with strong couplings at the smallest bosonic mass scales. In particular, the smallest stable zero of the interaction energy of the $2 A$ string yields a very precise prediction of the strong coupling at the $W$ mass scale, which, if evolved to the $Z^{0}$ scale, corresponds to the prediction $\alpha_{s}\left(m_{Z^{0}}\right)=0.117804(12)$. The current experimentally measured value is $\alpha_{s}\left(m_{Z^{0}}\right)=0.1185(20)$ [9].

Besides the coupling strengths of the three interactions, also the fermion mass spectrum can be obtained with high precision from chaotic strings. Here the expectation of the self energy of the chaotic strings is the relevant observable. One observes a large number of string couplings that locally minimize the self energy and at the same time numerically coincide with various running electroweak, strong, Yukawa and gravitational couplings, evaluated at the mass scales of the higher fermion families. The highest precision predictions for fermion masses comes from the self energy of the $2 A$ and $2 B$ strings, which is observed to exhibit minima for string couplings that coincide with gravitational and Yukawa couplings of all known fermions. The spectrum of these strings yields the free masses of the six quarks as $m_{u}=5.07(1) \mathrm{MeV}, m_{d}=9.35(1) \mathrm{MeV}, m_{s}=164.4(2) \mathrm{MeV}, m_{c}=1.259(4)$ $\mathrm{GeV}, m_{b}=4.22(2) \mathrm{GeV}$ and $m_{t}=164.5(2) \mathrm{GeV}$. Note that a free top 
mass prediction of $164.5(2) \mathrm{GeV}$ corresponds to a top pole mass prediction of $174.4(3) \mathrm{GeV}$, in very good agreement with the experimentally measured value $M_{t}=174.3 \pm 5.1 \mathrm{GeV}$. The masses of the charged leptons come out as $m_{e}=0.5117(8) \mathrm{MeV}, m_{\mu}=105.6(3) \mathrm{MeV}$ and $m_{\tau}=1.782(7) \mathrm{GeV}$. All these theoretically obtained values of fermion masses are in perfect agreement with experimental measurements. To the best of our knowledge, there is no other theoretical model that has achieved theoretical predictions of similar precision. Chaotic strings also provide evidence for massive neutrinos, and yield concrete predictions for the masses of the neutrino mass eigenstates $\nu_{1}, \nu_{2}, \nu_{3}$. These are $m_{\nu_{1}}=1.452(3) \cdot 10^{-5} \mathrm{eV}, m_{\nu_{2}}=2.574(3) \cdot 10^{-3} \mathrm{eV}$, $m_{\nu_{3}}=4.92(1) \cdot 10^{-2} \mathrm{eV}$.

Not only fermion masses, but also boson masses can be obtained from chaotic strings. The $2 \mathrm{~A}$ string correctly reproduces the masses of the $W$ and $Z$ boson, and a suitable interpretation of the $2 B^{-}$string dynamics provides evidence for the existence of a scalar particle of mass $m_{H}=154.4(5) \mathrm{GeV}$, which could be identified with the Higgs particle. The latter mass prediction is slightly larger than supersymmetric expectations but well within the experimental bounds based on the ordinary standard model. We also obtain estimates of the lightest glueball masses, which are consistent with estimates from lattice QCD.

This paper is organized as follows. In section 2 we introduce chaotic strings. In section 3 we write down (formal) potentials for the strings and define two types of vacuum energies, the interaction energy $W(a)$ and the selfenergy $V(a)$ of the chaotic string. Essentially $W(a)$ fixes coupling constants and $V(a)$ masses and mass mixing angles. In section 4 we introduce a physical interpretation of the chaotic string dynamics in terms of rapidly fluctuating virtual momenta. In sections 5 we will consider the analogue of an Einstein equation which makes arbitrary couplings evolve to the stable zeros of the interaction energy of the strings. We will provide numerical evidence that the smallest stable zeros of the $N=3$ strings numerically coincide with the electroweak coupling constants at the smallest fermionic mass scales. We then turn to $N=2$ strings and provide numerical evidence that the smallest stable zeros coincide with strong couplings at the smallest bosonic mass scales. In section 6 we will consider a suitable scalar field equation and provide evidence that local minima of the self-energy of the chaotic strings correctly reproduce the masses of all higher fermion families. We extract the fermion masses from the self energy of the $2 A, 2 B$ strings, as well as the boson masses from the $2 A^{-}, 2 B^{-}$strings. Our concluding remarks are given 
in section 7 .

\section{Chaotic Strings}

Chaotic strings are motivated by the assumption that there is a small-scale dynamics underlying the noise fields of the Parisi-Wu approach of stochastic quantization [7]. Since in string theory ordinary particles are believed to have string-like structure, it is natural to assume that also the noise used for second quantization in the Parisi-Wu approach may have such a string-like structure on a very small scale.

Among the many models that can be chosen to generate a deterministic chaotic noise string dynamics on a small scale certain criteria should be applied to select a particular system. First of all, for vanishing spatial coupling of the chaotic 'noise' one wants to have strongest possible random behavior with least possible higher-order correlations, in order to be closest to the Gaussian limit case (which corresponds to ordinary path integrals on a large scale). This selects as a local dynamics Tchebyscheff maps $T_{N}(x)$ of $N$-th order $(N \geq 2)$ [10]. It is well known that Tchebyscheff maps satisfy a Central Limit Theorem which guarantees the convergence to the Wiener process (and hence to ordinary path integrals) if sums of iterates are looked at from large scales [11, 12]. As shown in [10] Tschebyscheff maps have least higherorder correlations among all systems conjugated to a Bernoulli shift, and are in that sense closest to Gaussian white noise, though being completely deterministic. A graph theoretical method for this type of noise has been developed in 10, 13.

Moreover, when spatially coupling the discrete chaotic dynamics this should formally result from a Laplacian coupling rather than some other coupling, since this is the most relevant coupling form in quantum field and string theories. This leads to coupled map lattices of the diffusive coupling form. The resulting coupled map lattices can then be studied on lattices of arbitrary dimension (as done in [14]), but motivated by the fact that ordinary strings are 1-dimensional objects we will focus in this paper on the 1-dimensional case. One obtains a 'chaotic string' defined by

$$
\Phi_{n+1}^{i}=(1-a) T_{N}\left(\Phi_{n}^{i}\right)+s \frac{a}{2}\left(T_{N}^{b}\left(\Phi_{n}^{i-1}\right)+T_{N}^{b}\left(\Phi_{n}^{i+1}\right)\right) .
$$

$\Phi_{n}^{i}$ is a discrete chaotic noise field variable taking continuous values on the interval $[-1,1]$. The initial values $\Phi_{0}^{i}$ are randomly distributed in this in- 
terval. $i$ is a spatial lattice coordinate and $n$ a discrete time coordinate (in our case identified with the fictitious time of the Parisi-Wu approach). $T_{N}$ denotes the $N$-th order Tchebyscheff polynomial. In the following we will mainly study $T_{2}(\Phi)=2 \Phi^{2}-1$ and $T_{3}(\Phi)=4 \Phi^{3}-3 \Phi$. We consider both the positive and negative Tchebyscheff polynomial $T_{N}^{ \pm}(\Phi)= \pm T_{N}(\Phi)$, but have suppressed the index \pm in the above equation. The variable $a$ is a coupling constant taking values in the interval $[0,1]$. Since $a$ determines the strength of the Laplacian coupling, $a^{-1}$ can be regarded as a kind of metric in the 1-dimensional string space indexed by i. $s$ is a sign variable taking on the values \pm 1 . The choice $s=+1$ is called 'diffusive coupling', but for symmetry reasons it also makes sense sense to study the choice $s=-1$, which we call 'anti-diffusive coupling'. The integer $b$ distinguishes between the forward and backward coupling form [14], $b=1$ corresponds to forward coupling $\left(T_{N}^{1}(\Phi):=T_{N}(\Phi)\right), b=0$ to backward coupling $\left(T_{N}^{0}(\Phi):=\Phi\right)$. We consider periodic boundary conditions and large lattices of size $i_{\max }$.

One can easily check that for odd $N$ the choice of $s$ is irrelevant (since odd Tchebyscheff maps satisfy $T_{N}(-\Phi)=-T_{N}(\Phi)$ ), whereas for even $N$ the sign of $s$ is relevant and a different dynamics arises. Hence, restricting ourselves to $N=2$ and $N=3$, in total 6 different chaotic string theories arise, characterized by $(N, b, s)=(2,1,+1),(2,0,+1),(2,1,-1),(2,0,-1)$ and $(N, b)=(3,1),(3,0)$. For easier notation, in the following we will label these string theories as $2 A, 2 B, 2 A^{-}, 2 B^{-}, 3 A, 3 B$, respectively.

If the coupling $a$ is sufficiently small, the chaotic variables $\Phi_{n}^{i}$ can be used to generate the noise of the Parisi-Wu approach of stochastic quantization on a very small scale. It can actually be shown that if chaotic Tchebyscheff noise is coupled to a slowly varying dynamics, in first and second order perturbation theory the cases $N>3$ do not yield anything new compared to Gaussian white noise (see [8] for more details). Hence the above six chaotic string theories obtained for $N=2$ and $N=3$ are the most relevant ones to consider, yielding non-trivial behaviour in leading order of stochastic quantization.

As shown in [7, 14], the chaotic noise string dynamics (11) formally originates from a 1-dimensional continuum $\phi^{N+1}$-theory in the limit of infinite self-interaction strength (see Appenidix A for deriving, as an example, the $3 B$ string dynamics). In this sense, chaotic strings can also be regarded as degenerated Higgs-like fields with infinite self-interaction parameters, which are constraint to a 1-dimensional space. 


\section{$3 \quad$ Vacuum energy of chaotic strings}

Though the chaotic string dynamics is dissipative, one can formally introduce potentials that generate the discrete time evolution. For $a=0$ we may write

$$
\Phi_{n+1}-\Phi_{n}= \pm T_{N}\left(\Phi_{n}\right)=-\frac{\partial}{\partial \Phi_{n}} V_{ \pm}\left(\Phi_{n}\right)
$$

For $N=2$ the (formal) potential is given by

$$
V_{ \pm}^{(2)}(\Phi)= \pm\left(-\frac{2}{3} \Phi^{3}+\Phi\right)+\frac{1}{2} \Phi^{2}+C
$$

for $N=3$ by

$$
V_{ \pm}^{(3)}(\Phi)= \pm\left(-\Phi^{4}+\frac{3}{2} \Phi^{2}\right)+\frac{1}{2} \Phi^{2}+C
$$

Here $C$ is an arbitrary constant. The uncoupled case $a=0$ is completely understood. The dynamics is ergodic and mixing. Any expectation of an observable $A(\Phi)$ can be calculated as

$$
\langle A\rangle=\int_{-1}^{1} d \phi \rho(\phi) A(\phi)
$$

where

$$
\rho(\phi)=\frac{1}{\pi \sqrt{1-\phi^{2}}},
$$

is the natural invariant density describing the probability distribution of iterates of Tchebyscheff maps (see, e.g. [15]). In the formalism of nonextensive statistical mechanics [16], this probability density can be regarded as a generalized canonical distribution with entropic index $q=3$, or as an escort distribution with index $q=-1$.

If a spatial coupling $a$ is introduced, things become much more complicated, and the invariant 1-point density deviates from the simple form (6). A spatial coupling is formally generated by the interaction potential $a W_{ \pm}(\Phi, \Psi)$, with

$$
W_{ \pm}(\Phi, \Psi)=\frac{1}{4}(\Phi \pm \Psi)^{2}+C .
$$

Here $\Phi$ and $\Psi$ are neighboured noise field variables on the lattice. One has

$$
-\frac{\partial}{\partial \Phi^{i}} W_{ \pm}\left(\Phi^{i}, \Phi^{i+1}\right)-\frac{\partial}{\partial \Phi^{i}} W_{ \pm}\left(\Phi^{i}, \Phi^{i-1}\right)= \pm \frac{1}{2} \Phi^{i+1}-\Phi^{i} \pm \frac{1}{2} \Phi^{i-1}
$$


This generates diffusive $(+)$, respectively anti-diffusive $(-)$ coupling. Antidiffusive coupling can equivalently be obtained by keeping $W_{-}$but replacing $T_{N} \rightarrow-T_{N}$ at odd lattice sites. The coupled map dynamics (11) is obtained by letting the action of $V$ and $W$ alternate in discrete time $n$, then regarding the two time steps as one. For the backward coupling form $b=0$, the action of the potentials also alternates in discrete space $i$.

The expectations of the potentials $V$ and $W$ yield two types of vacuum energies $V(a):=\left\langle V_{ \pm}^{(N)}\left(\Phi^{i}\right)\right\rangle$ (the self energy) and $W(a):=\left\langle W_{ \pm}\left(\Phi^{i}, \Phi^{i+1}\right)\right\rangle$ (the interaction energy). Here $\langle\cdots\rangle$ denotes the expectation with respect to the coupled chaotic dynamics. Numerically, any such expectation can be determined by averaging over all $i$ and $n$ for random initial conditions $\Phi_{0}^{i} \in[-1,1]$, omitting the first few transients. Note that in the stochastic quantization approach the chaotic noise is used for 2nd quantization of standard model fields (or ordinary strings) via the Parisi-Wu approach [7]. Hence generally expectations with respect to the chaotic dynamics correspond to expectations with respect to 2 nd quantization. The expectation of the vacuum energy of the string, given by the above functions $W(a)$ and $V(a)$, depends on the coupling $a$ in a non-trivial way. Moreover, it also depends on the integers $N, b, s$ that define the chaotic string theory.

Since negative and positive Tchebyscheff maps essentially generate the same dynamics, up to a sign, any physically relevant observable should be invariant under the transformation $T_{N} \rightarrow-T_{N}$. The vacuum energies $V(a)$ and $W(a)$ of the various strings exhibit full symmetry under the transformation $T_{N} \rightarrow-T_{N}$ (respectively $s \rightarrow-s$ ) if the additive constant $C$ is chosen to be

$$
C=-\frac{1}{2}\left\langle\Phi^{2}\right\rangle
$$

For that choice of $C$, the expectations of $V_{+}$and $V_{-}$as well as those of $W_{-}$ and $W_{+}$are the same, up to a sign.

Choosing (by convention) the + sign one obtains from eq. (3), (4) and (17) for the expectations of the potentials

$$
\begin{aligned}
V^{(2)}(a) & =-\frac{2}{3}\left\langle\Phi^{3}\right\rangle+\langle\Phi\rangle \\
V^{(3)}(a) & =-\left\langle\Phi^{4}\right\rangle+\frac{3}{2}\left\langle\Phi^{2}\right\rangle,
\end{aligned}
$$

and

$$
W(a)=\frac{1}{2}\left\langle\Phi^{i} \Phi^{i+1}\right\rangle .
$$


The above \pm symmetry can actually be used to cancel unwanted vacuum energy and to avoid problems with the cosmological constant. If one assumes that strings with both $T_{N}$ and $-T_{N}$ are physically relevant, the two contributions $V(a)$ and $-V(a)$ (respectively $W(a)$ and $-W(a)$ ) may simply add up to zero. This reminds us of similarly good effects that supersymmetric partners have in ordinary quantum field and string theories. As a working hypothesis, we will thus formally associate the above symmetry with supersymmetry in the chaotic noise space.

Similarly as for ordinary strings it also makes sense to consider certain conditions of constraints for the chaotic string. For ordinary strings (for example bosonic strings in covariant gauge [1]) one has the condition of constraint that the energy momentum tensor should vanish. The first diagonal component of the energy momentum tensor is an energy density. For chaotic strings, the evolution in space $i$ is governed by the potential $W_{ \pm}(\Phi, \Psi)$ and the corresponding expectation of the energy density is $\pm W(a)$. We should thus impose the condition of constraint that $W(a)$ should vanish for physically observable states. Moreover, the evolution in fictitious time $n$ is governed by the self-interacting potential $V^{(N)}(\Phi)$. This potential generates a shift of information, since the Tchebyscheff maps $T_{N}$ are conjugated to a Bernoulli shift of $N$ symbols. Hence $V(a)$ can be regarded as the expectation of a kind of information potential or entropy function, which, motivated by thermodynamics, should be extremized for physically observable states. Note that the action of $V$ and $W$ alternates in $n$ and $i$ direction. Both types of vacuum energies describe different relevant observables of the chaotic string and are of equal importance.

\section{Physical interpretation of the chaotic string dynamics in terms of vacuum fluctuations}

In order to construct a link to standard model phenomenology it is useful to introduce a simple physical interpretation of the chaotic string dynamics in

terms of fluctuating virtual momenta. Suppose we regard $\Phi_{n}^{i}$ to be a fluctuating virtual momentum component associated with a hypothetical particle $i$ at (fictitious) time $n$ that lives in the constraint 1-dimensional string space. Then neighboured particles $i$ and $i-1$ exchange momenta due to the diffusive coupling. A more detailed physical interpretation would be that at each time 
step $n$ a particle-antiparticle pair $f_{1}, \bar{f}_{2}$ is being created in cell $i$ by the field energy of the self-interacting potential. In units of some arbitrary energy scale $p_{\max }$, the particle has momentum $\Phi_{n}^{i}$, the antiparticle momentum $-\Phi_{n}^{i}$. They interact with particles in neighboured cells by exchange of a (hypothetical) gauge boson $B_{2}$, then they annihilate into another boson $B_{1}$ and the next chaotic vacuum fluctuation (the next creation of a particle-antiparticle pair) takes place. This can be symbollically described by the Feynman graph in Fig. 1. Actually, the graph continues ad infinitum in time and space and could thus be called a 'Feynman web', since it describes an extended spatiotemporal interaction state of the string, to which we have given a standard model-like interpretation. The important point is that in this interpretation $a$ is a (hypothetical) standard model coupling constant, since it describes the strength of momentum exchange of neighboured particles. At the same time, $a$ can also be regarded as an inverse metric in the 1-dimensional string space, since it determines the strength of the Laplacian coupling.

It is well known that standard model interaction strengths actually depend on the relevant energy scale $E$. We have the running electroweak and strong coupling constants. What should we now take for the energy (or temperature) $E$ of the chaotic string? A priori this is unknown. However, we will present extensive numerical evidence that the constraints on the vacuum energy of the chaotic string fix certain discrete string couplings $a_{i}$, and these string couplings are numerically observed to coincide with running standard model couplings, the energy (or temperature) being given by

$$
E=\frac{1}{2} N\left(m_{B_{1}}+m_{f_{1}}+m_{f_{2}}\right)
$$

Here $N$ is the index of the chaotic string theory considered, and $m_{B_{1}}, m_{f_{1}}, m_{f_{2}}$ denote the masses of the particles involved in the Feynman web interpretation. The surprising observation is that rather than yielding just some unknown exotic physics, the chaotic string spectrum appears to reproduce the masses and coupling constants of the known quarks, leptons and gauge bosons of the standard model (plus possibly more) [2.

Formula (13) formally reminds us of the energy levels $E_{N}=\frac{N}{2} \hbar \omega$ of a quantum mechanical harmonic oscillator, with low-energy levels $(N=2,3)$ given by the masses of the standard model particles. In the Feynman web

\footnotetext{
${ }^{2}$ If the presence of black holes is assumed, one can also relate $E$ to the Hawking temperature $k T_{H} \sim \frac{1}{G M}$, thus connecting very large black hole masses $M$ with very small temperatures $k T_{H}$.
} 
interpretation of Fig. 1, the formula is plausible. We expect the process of Fig. 1 to be possible as soon as the energy per cell $i$ is of the order $m_{B_{1}}+$ $m_{f_{1}}+m_{f_{2}}$. The boson $B_{2}$ is virtual and does not contribute to the energy scale. The factor $N$ can be understood as a multiplicity factor counting the degrees of freedom. Given some value $\Phi_{n}^{i}$ of the momentum in cell $i$, there are $N$ different pre-images $T_{N}^{-1}\left(\Phi_{n}^{i}\right)$ how this value of the momentum can be achieved. All these different channels contribute to the energy scale.

\section{Zeros of the interaction energy $W(a)$}

\subsection{Analogue of the Einstein field equations}

Let us now suppose that a priori arbitrary standard model couplings $\alpha$ are possible. Assume that these couplings are at the same time coupling constants $a$ in the chaotic string space. In fact, we may then regard $\alpha^{-1}=a^{-1}$ as a kind of metric in the 1-dimensional string space. A stochastically quantized Einstein equation for this 1-dimensional (trivial) metric in the 1-dimensional string space would then have the form

$$
\left(\frac{1}{a}\right)=-\frac{1}{a^{2}} \dot{a}=T_{00}+\text { noise. }
$$

Here $T_{00}$ is the energy density of the vacuum as created by the interaction energy $W$ of the chaotic strings, and $t$ is the fictitious time of the Parisi-Wu approach, used for second quantization. Choosing $T_{00}=-\Gamma \cdot W$, where $\Gamma$ is a positive constant, one ends up with

$$
\dot{a}=a^{2} \Gamma W(a)+\text { noise. }
$$

The fictitious time $t$ of the Parisi-Wu approach has dimension energy ${ }^{-2}$, hence if $W(a)$ is defined to be dimensionless then the constant $\Gamma$ should have dimension energy ${ }^{2}$, and we could for example choose $\Gamma \sim G^{-1}$ or $\Gamma \sim$ $a^{-2} G^{-1}$, where $G$ is the gravitational constant.

Apparently all zeros of the function $W(a)$ describe a stationary state for possible standard model couplings, but only those zeros where $W(a)$ has locally negative slope decribe a stable stationary state. Arbitrary initial couplings $a$ will evolve to these stable states (Fig. 2). Of course the stability properties depend on the choice of the sign of the constant $\Gamma$. This choice 
breaks the initial symmetry of the problem. Let us assume that for our world $\Gamma>0$ is relevant and label the stable zeros of $W(a)$ (i.e. those with negative slope) by $a_{i}^{(N b)}, i=1,2, \ldots$. Here $N$ is the index of the string theory considered, and $b=1,0$ (or A,B) distinguishes between forward and backward coupling. We will now describe our numerical findings in detail.

\subsection{The 3A string - electric interaction strengths of electrons and $d$-quarks}

Fig. 3 shows the interaction energy $W(a)=\frac{1}{2}\left\langle\Phi_{n}^{i} \Phi_{n}^{i+1}\right\rangle$ of the chaotic $3 A$ string. We observe the following stable zeros in the low-coupling region $a \in[0,0.018]:$

$$
\begin{aligned}
& a_{1}^{(3 A)}=0.0008164(8) \\
& a_{2}^{(3 A)}=0.0073038(17)
\end{aligned}
$$

The statistical error is estimated by repeating the iteration of the coupled map lattice (11) for different random initial conditions $\Phi_{0}^{i} \in[-1,1]$. We used coupled map lattices of size 10000 with periodic boundary conditions, typical iteration times over which the product $\Phi_{n}^{i} \Phi_{n}^{i+1}$ was averaged were $n_{\max }=10^{7}$.

Remarkably, the zero $a_{2}^{(3 A)}$ appears to approximately coincide with the fine structure constant $\alpha_{e l} \approx 1 / 137$. To construct a suitable Feynman web interpretation, let us choose in Fig. 1 for $B_{1}$ any massless boson and $B_{2}=\gamma$, $f_{1}=e^{-}, \bar{f}_{2}=e^{+}$. The relevant energy scale underlying this Feynman web is given by $E=(3 / 2)\left(m_{\gamma}+2 m_{e}\right)=3 m_{e}$, according to eq. (13). Hence our standard model interpretation of the string state described by $a_{2}^{(3 A)}$ suggests the numerical identity

$$
a_{2}^{(3 A)}=\alpha_{e l}\left(3 m_{e}\right) .
$$

For a precise numerical comparison let us estimate the running electromagnetic coupling at this energy scale. We may use the 1st-order QED formula

$\alpha_{e l}(E)=\alpha_{e l}(0)\left\{1+\frac{2 \alpha_{e l}(0)}{\pi} \sum_{i} f_{i} \int_{0}^{1} d x x(1-x) \log \left(1+\frac{E^{2}}{m_{i}^{2}} x(1-x)\right)\right\}$,

The sum is over all charged elementary particles, $m_{i}$ denotes their (free) masses, and $f_{i}$ are charge factors given by 1 for $e, \mu, \tau$-leptons, $\frac{4}{3}$ for $u, c, t$ quarks and $\frac{1}{3}$ for $d, s, b$-quarks. Using this formula, we get $\alpha_{e l}\left(3 m_{e}\right)=$ 
0.007303 , to be compared with $a_{2}^{(3 A)}=0.0073038(17)$. There is excellent agreement.

Next, we notice that the zero $a_{1}^{(3 A)}$ has approximately the value $\frac{1}{9} \alpha_{e l}$. This could mean that the chaotic $3 \mathrm{~A}$ string also has a mode that provides evidence for electrically interacting $d$-quarks. Our interpretation is

$$
a_{1}^{(3 A)}=\alpha_{e l}^{d}\left(3 m_{d}\right)=\frac{1}{9} \alpha_{e l}\left(3 m_{d}\right)
$$

where $\alpha_{e l}^{d}=\frac{1}{9} \alpha_{e l}$ denotes the electromagnetic interaction strength of $d$ quarks. In the Feynman web interpretation, we have $B_{1}$ massless, $B_{2}=\gamma$, $f_{1}=d, f_{2}=\bar{d}$. Formula (17), as an estimate, yields for $m_{d}=9 \mathrm{MeV}$ the value $\alpha_{e l}\left(3 m_{d}\right)=0.007349$, which coincides very well with $9 a_{1}^{(3 A)}=0.007348(7)$. The value $9 a_{1}^{(3 A)}$ actually translates to the energy scale $E_{d}=(26.0 \pm 6.4)$ $\mathrm{MeV}$. This yields $m_{d}=\frac{1}{3} E_{d}=(8.7 \pm 2.1) \mathrm{MeV}$, which coincides with estimates of the $\overline{M S}$ current quark mass of the $d$ quark at the proton mass renormalization scale 9.

\subsection{The 3B string - weak interaction strengths of neu- trinos and $u$-quarks}

The 3B string is a system with backward coupling. For backward coupling the action of the potentials $V$ and $W$ not only alternates in time $n$ but also in discrete space $i$. Hence one may conjecture that this coupling form generally describes mixed states of two particles.

The interaction energy $W(a)$ of the $3 \mathrm{~B}$ string is plotted in Fig. 4 . In the low-coupling region $a \in[0,0.018]$ we observe the following stable zeros of $W(a)$ :

$$
\begin{aligned}
& a_{1}^{(3 B)}=0.0018012(4) \\
& a_{2}^{(3 B)}=0.017550(1)
\end{aligned}
$$

If our approach is consistent, we might be able to find an interpretation of $a_{1}^{(3 B)}$ and $a_{2}^{(3 B)}$ indicating the existence of $u$-quarks and neutrinos.

Let us start with $a_{2}^{(3 B)}$. For left-handed neutrinos, the weak coupling due to the exchange of $Z^{0}$-bosons is given by

$$
\alpha_{w e a k}^{\nu_{L}}=\alpha_{e l} \frac{1}{4 \sin ^{2} \theta_{W} \cos ^{2} \theta_{W}} .
$$


Here $\theta_{W}$ is the weak mixing angle. In the following we will treat $\sin ^{2} \theta_{W}$ as an effective constant, and regard $\alpha_{e l}$ as the running electromagnetic coupling. Other renormalization schemes are also possible, but yield only minor numerical differences. Experimentally, at LEP the effective weak mixing angle is measured as $\sin ^{2} \theta_{W}=0.2318(2)$ [9]. Assuming that in addition to the left-handed neutrino interacting weakly there is an electron interacting electrically, the two interaction processes can add up independently if the electron is right-handed, since right-handed electrons cannot interact with left-handed neutrinos. Hence a possible standard model interpretation of the zero $a_{2}^{(3 B)}$ would be

$$
a_{2}^{(3 B)}=\alpha_{e l}\left(3 m_{e}\right)+\alpha_{w e a k}^{\nu_{L}}\left(3 m_{\nu_{e}}\right)=a_{2}^{(3 A)}+\alpha_{e l}\left(3 m_{\nu_{e}}\right) \frac{1}{4 \sin ^{2} \theta_{W} \cos ^{2} \theta_{W}}
$$

In the Feynman web interpretation of Fig. $1, B_{2}=Z^{0}, f_{1}=\nu_{L}, \overline{f_{2}}=\overline{\nu_{L}}$ in addition to the process already described by $a_{2}^{(3 A)}$. $B_{1}$ can again be any massless boson. Putting in the experimentally measured value of $\sin ^{2} \theta_{W}=$ 0.2318 , we obtain for the right-hand side of eq. (20) the value 0.01755 , which coincides perfectly with the observed string coupling $a_{2}^{(3 B)}=0.017550$.

Next, let us interpret $a_{1}^{(3 B)}$. In analogy to the joint appearance of $\nu$ and $e$, we should also expect to find a weakly interacting $u$-quark, together with a $d$-quark interacting electrically. Clearly, the $u$-quark could also interact electrically, but for symmetry reasons we expect the pair $(u, d)$ to interact in a similar way as $(\nu, e)$. A right-handed $u$-quark interacts weakly with the coupling

$$
\alpha_{w e a k}^{u_{R}}=\frac{4}{9} \alpha_{e l} \frac{\sin ^{2} \theta_{W}}{\cos ^{2} \theta_{W}}
$$

Adding up the electrical interaction strength of a $d$-quark, a natural interpretation, quite similar to that of the zero $a_{2}^{(3 B)}$, is

$$
a_{1}^{(3 B)}=\alpha_{e l}^{d}\left(3 m_{d}\right)+\alpha_{w e a k}^{u_{R}}\left(3 m_{u}\right)=a_{1}^{(3 A)}+\frac{4}{9} \alpha_{e l}\left(3 m_{u}\right) \frac{\sin ^{2} \theta_{W}}{\cos ^{2} \theta_{W}}
$$

The Feynman web interpretation of this string state is $B_{1}$ massless, $B_{2}=Z^{0}$, $f_{1}=u_{R}, f_{2}=\overline{u_{R}}$ in addition to the process underlying $a_{1}^{(3 A)}$. Numerically, taking $\sin ^{2} \theta_{W}=0.2318$ and evaluating the running $\alpha_{e l}$ using $m_{u}=5$ $\mathrm{MeV}$, we obtain for the right-hand side of eq. (22) 0.001800, which should be compared with $a_{2}^{(3 B)}=0.001801$. Again we have perfect agreement within 
the first 4 digits. It is remarkable that the same universal effective value $\sin ^{2} \theta_{W}=0.2318$ can be used consistently for both leptons (couplings $a_{2}^{(3 A)}$, $a_{2}^{(3 B)}$ ) and quarks (couplings $a_{1}^{(3 A)}, a_{1}^{(3 B)}$ ). If, on the other hand, we assume that $\sin ^{2} \theta_{W}$ is fixed by $a_{2}^{(3 B)}$ to be 0.2318 , the running electric coupling can be used to extract the energy scale $E$ from $a_{1}^{(3 B)}$. We obtain $E=3 m_{u}=(21.3 \pm 9) \mathrm{MeV}$, meaning $m_{u}=(7.1 \pm 3.0) \mathrm{MeV}$.

Note that generally the backward coupling form of the $N=3$ strings seems to describe a spinless state (formed by $e_{R}$ and $\nu_{L}$, respectively $d_{L}$ and $u_{R}$ ), whereas the forward coupling form just describes one particle species with non-zero spin $(e$ or $d)$. A similar statement will turn out to hold for the $N=2$ theories, replacing fermions by bosons.

\subsection{High-precision prediction of the electroweak pa- rameters}

It appears that the smallest stable zeros of the $N=3$ strings coincide with the electroweak coupling strengths at the mass scales of the lightest fermions $d, u, e, \nu_{e}$. We can thus actually invert the formulas and give a rather precise prediction of the electroweak parameters. The general idea underlying this is that at some early stage of the universe (possibly in a pre-big bang scenario) the electroweak couplings were not fixed but were following the evolution equation (15), being finally fixed as stable inverse metrics in the string space. The chaotic string dynamics may still persist today and stabilize the observed parameter values. The observed low-energy limit of the fine structure constant follows from $a_{2}^{(3 A)}$ and formula (17) as

$$
\begin{aligned}
\alpha_{e l}(0) & =a_{2}^{(3 A)}\left(1-\frac{2 a_{2}^{(3 A)}}{\pi} \sum_{i} f_{i} \int_{0}^{1} d x x(1-x) \log \left(1+\frac{9 m_{e}^{2}}{m_{i}^{2}} x(1-x)\right)\right) \\
& =0.0072979(17)=1 / 137.03(3) .
\end{aligned}
$$

Here we used the known value of the electron mass and neglected terms of order $\alpha^{3}$, which are much smaller than the statistical error. Within the error bounds, our result agrees very well with the experimentally measured value $\alpha(0)=0.00729735=1 / 137.036$ (the experimentally measured number is known to quite precisely coincide with $\frac{1}{137} \cdot \cos \frac{\pi}{137}=0.00729735$ [17]).

Next, the effective weak mixing angle can be predicted from eq. (20). 
Since $m_{\nu_{e}} \approx 0$ one obtains

$$
\sin ^{2} \theta_{W}=\frac{1}{2}\left(1-\sqrt{1-\frac{\alpha(0)}{a_{2}^{(3 B)}-a_{2}^{(3 A)}}}\right)
$$

This yields

$$
\sin ^{2} \theta_{W}=0.23177(7) .
$$

The value perfectly coincides with experimental measurements [9]. In fact, the prediction is much more precise than the supersymmetric GUT model prediction $0.234(2)$. Within the error bars, we actually do get the same effective Weinberg angle for quarks and leptons using either $a_{1}^{(3 B)}$ or $a_{2}^{(3 B)}$.

Any high-precision result on $\sin ^{2} \theta_{W}$ can also be used to estimate gauge and Higgs boson masses from radiative corrections. For example we may use the results of a calculation by Degrassi et al. [18]. Our Feynman web interpretation of the zero $a_{2}^{(2 B)}$ suggests to regard our $\sin ^{2} \theta_{W}$ as the effective mixing parameter $\bar{s}_{l}^{2}=\sin ^{2} \theta_{\text {eff }}^{\text {lept }}$ for $Z^{0}$-lepton coupling. Table 1 in [18] translates the value $0.23177(7)$ to $m_{W}=80.35(1) \mathrm{GeV}$ and yields a Higgs mass of the order of twice the $W$ mass.

Note that there is excellent agreement of the weak mixing angle obtained from the chaotic 2B string with the experimental measurements at LEP. At LEP the effective weak mixing angle $\bar{s}_{l}^{2}$ is measured as 0.23185(23), from this the Higgs mass is estimated as $166_{-95}^{+270} \mathrm{GeV}$ and the W-mass as 80.35(6) $\mathrm{GeV}$ [9].

\subsection{The 2A string - strong interaction strength at the $W$-mass scale}

If electroweak coupling strengths are fixed by suitable zeros of the interaction energy of chaotic strings, then something similar should also be the case for the strong coupling strength $\alpha_{s}$. Let us now look at strings with $N=2$. Fig. 5 shows the interaction energy $W(a)$ of the $2 \mathrm{~A}$ string. Only one stable zero is observed:

$$
a_{1}^{(2 A)}=0.120093(3)
$$

We notice that it numerically seems to coincide with the strong coupling constant $\alpha_{s}$ at the $W$ - or $Z$ mass scale, which is experimentally measured as $\alpha_{s}\left(m_{Z}\right)=0.1185(20)[9]$. 
For symmetry reasons, it seems plausible that if the $N=3$ strings fix the electroweak couplings at the smallest fermionic mass scales, then $N=2$ strings could fix the strong couplings at the smallest bosonic mass scales. The lightest massive gauge boson is indeed the $W^{ \pm}$. Hence our Feynman web interpretation associated with $a_{1}^{(2 A)}$ is $B_{1}=W^{ \pm}, B_{2}=g$ (gluon), $f_{1}=u$, $\bar{f}_{2}=\bar{d}$ (respectively $f_{1}=d, \bar{f}_{2}=\bar{u}$ ), and since $N=2$ formula (13) implies

$$
a_{1}^{(2 A)}=\alpha_{s}\left(m_{W}+m_{u}+m_{d}\right) \approx \alpha_{s}\left(m_{W}\right) .
$$

Since the $W$-mass is known with high precision, eq. (27) yields quite a precise prediction for the strong coupling $\alpha_{s}$. We can evolve it to arbitrary energy scales, using the well known perturbative results from QCD (see Appendix B). In 3rd-order perturbation theory eq. (27) is equivalent to an effective QCD scale parameter

$$
\Lambda^{(5)}=0.20608(14) G e V,
$$

neglecting higher-order terms. Here we use for the $W$-mass the value measured at LEP, $m_{W}=80.35(6) \mathrm{GeV}$ [9]. At $E=m_{Z}=91.188(2) \mathrm{GeV}$ eq. (111) in Appendix B yields

$$
\alpha_{s}\left(m_{Z^{0}}\right)=0.117804(12) .
$$

This prediction of $\alpha_{s}$ from the zero of the chaotic 2A string is clearly consistent with the experimentally measured value $0.1185(20)$ and in fact much more precise than current experiments can verify.

To evolve $\alpha_{s}(E)$ to energies $E>M_{t}$ or $E<M_{b}$ one has to take into account quark threshold effects. In chapter 6 we will obtain from the self energy of the chaotic strings the free bottom and top masses as $m_{b}=4.23(1) \mathrm{GeV}$ and $m_{t}=164.5(2) \mathrm{GeV}$. The corresponding pole masses are $M_{b}=4.87(2)$ $\mathrm{GeV}$ and $M_{t}=174.3(3) \mathrm{GeV}$ (formula (115) in Appendix B). Matching the running $\alpha_{s}(E)$ in a continuous way at $M_{t}$ and $M_{b}$ [19], we obtain from the above value $\Lambda^{(5)}=0.20608(14) \mathrm{GeV}$ the values $\Lambda^{(6)}=0.08705(6) \mathrm{GeV}$ and $\Lambda^{(4)}=0.28913(17) \mathrm{GeV}$. Slight numerical differences can arise from the way the quark thresholds are treated.

\footnotetext{
${ }^{3}$ see Appendix B for the definition of 'free' quark masses
} 


\subsection{The 2B string - the lightest scalar glueball}

The interaction energy of the $2 \mathrm{~B}$ string is shown in Fig. 6 . $W(a)$ has only one non-trivial zero

$$
a_{1}^{(2 B)}=0.3145(1)
$$

It has negative slope, so it should describe an observable stable state. One possibility is to interpret this as a strong coupling at the lightest glueball mass scale. The lightest scalar glueball has spin $J^{P C}=0^{++}$and is denoted by $g g^{0++}$ in the following. If we choose the Feynman web interpretation $\left.B_{1}=g g^{0++}, B_{2}=g, f_{1}=u, \bar{f}_{2}=\bar{u}\right)$, meaning

$$
a_{1}^{(2 B)}=\alpha_{s}\left(m_{g g^{0++}}+2 m_{u}\right) \approx \alpha_{s}\left(m_{g g^{0++}}\right),
$$

then indeed the $2 \mathrm{~B}$ string describes two bosons (two gluons forming a glueball), similar to the $3 \mathrm{~B}$ string, which described two fermions. In both cases a spin 0 state is formed. In lattice gauge calculations including dynamical fermions the smallest scalar glueball mass is estimated as $m_{g g^{0++}}=$ $(1.74 \pm 0.07) \mathrm{GeV} 20$ and at this energy the running strong coupling constant is experimentally measured to be $\alpha_{s}=0.32 \pm 0.05$ [21]. This clearly is consistent with the observed value of $a_{1}^{(2 B)}$.

We can estimate the lightest glueball mass predicted by the $2 \mathrm{~B}$ string using once again the 3rd-order QCD formula (111) in Appendix B. The value $\alpha_{s}\left(m_{g g^{0++}}+2 m_{u}\right)=0.3145(1)$ then translates to $m_{g g^{0++}}+2 m_{u}=1.812(2)$ $\mathrm{GeV}$.

\subsection{The $2 \mathrm{~A}^{-}$and $2 \mathrm{~B}^{-}$strings - towards a Higgs mass prediction}

Two chaotic string theories are still remaining, namely those with $N=2$ and antidiffusive coupling. The interaction energies $W(a)$ of the $2 \mathrm{~A}^{-}$and $2 \mathrm{~B}^{-}$ strings are shown in Fig. 7 and 8. Let us now try to find a suitable Feynman

web interpretation for the observed smallest stable zeros $a_{1}^{\left(2 A^{-}\right)}=0.1758(1)$ and $a_{1}^{\left(2 B^{-}\right)}=0.095370(1)$ of these strings.

Again let us be guided by symmetry considerations. We saw that the smallest stable zero of the $2 \mathrm{~A}$ string described a boson with non-zero spin (the lightest massive gauge boson $W^{ \pm}$) and the smallest stable zero of the 2B string a boson without spin (the lightest scalar glueball). Thus it seems reasonable to assume that the smallest stable zero of the $2 \mathrm{~A}^{-}$string describes 
yet another bosonic particle with non-zero spin, possibly the lightest glueball with spin $J^{P C}=2^{++}$, and the smallest zero of the $2 \mathrm{~B}^{-}$string yet another bosonic particle with spin 0, possibly the lightest Higgs boson.

Let us start with $a_{1}^{\left(2 A^{-}\right)}$. Our Feynman web interpretation of this string state is $B_{1}=g g^{2++}, B_{2}=g, f_{1}=q, \bar{f}_{2}=\bar{q}$, where $q, \bar{q}$ are suitable quarks. From the strong coupling interpretation

$$
a_{1}^{\left(2 A^{-}\right)}=\alpha_{s}\left(E_{1}\right)
$$

the energy $E_{1}=m_{g g^{2++}}+2 m_{q}$ can again be determined from the QCD formula (111), using our previously determined $\Lambda^{(5)}$. One obtains $E_{1}=10.45(3)$ $\mathrm{GeV}$. In lattice gauge calculations the mass of the lightest $2^{++}$glueball is estimated as 2.23(31) GeV [9]. We thus get the correct order of magnitude of the $2^{++}$glueball mass if we assume that the quarks in the Feynman web interpretation are bottom quarks. In this case a glueball mass $m_{g g 2^{++}}=(10.45(3)-2 \cdot 4.23(1)) \mathrm{GeV}=1.99(4) \mathrm{GeV}$ is predicted, using $m_{b}=4.23(1) \mathrm{GeV}$.

Next, let us consider $a_{1}^{\left(2 B^{-}\right)}$. This zero is even more interesting, since it may provide evidence for the Higgs particle. Our Feynman web interpretation is $B_{1}=H, B_{2}=g, f_{1}=q, \bar{f}_{2}=\bar{q}$, where $H$ is the lightest Higgs boson and $q, \bar{q}$ are suitable quarks. The strong coupling interpretation

$$
a_{1}^{\left(2 B^{-}\right)}=\alpha_{s}\left(E_{2}\right)
$$

yields $E_{2}=483.4(3) \mathrm{GeV}=m_{H}+2 m_{q}$. However, experimental and theoretical arguments [9] imply that the Higgs mass should be in the region 100...200 $\mathrm{GeV}$. Hence we only obtain a consistent value for the Higgs mass if we assume that the quarks involved are $t$ quarks. This is similar to the zero $a_{1}^{\left(2 A^{-}\right)}$, where the quarks involved were also heavy quarks. Generally, strings with antidiffusive couplings seem to describe heavy rather than light particles.

In section 6.5 we will obtain quite a precise prediction of the free top mass from the self energy of the chaotic $2 \mathrm{~B}$ string, namely $m_{t}=164.5(2) \mathrm{GeV}$, corresponding to a top pole mass of $174.4(3) \mathrm{GeV}$. With this value the zero $a_{1}^{\left(2 B^{-}\right)}$yields a Higgs mass prediction of

$$
m_{H}=E_{2}-2 m_{t}=154.4(5) \mathrm{GeV} .
$$

This is a very precise prediction, the statistical error is very small. But of course the main source of uncertainty is a theoretical uncertainty, namely 
whether our Feynman web interpretation of the zero $a_{1}^{\left(2 B^{-}\right)}$is correct. For example, assuming that a supersymmetric extension of the standard model is correct, then the zero could also describe another scalar particle.

Similar as for the $N=3$ strings, the next larger zeros $a_{2}^{\left(2 A^{-}\right)}$and $a_{2}^{\left(2 B^{-}\right)}$ should be considered as well. What is interesting here is the fact that near $a=\frac{1}{2}\left(2 A^{-}\right)$and near $a=1\left(2 B^{-}\right)$all chaotic fluctuations cease to exist, and the fields $\Phi_{n}^{i}$ approach a stable homogeneous fixed point 0 at $a_{2}^{\left(2 A^{-}\right)}=\frac{1}{2}$, respectively $a_{2}^{\left(2 B^{-}\right)}=1$. From a stochastic quantization point of view this means that there are no vacuum fluctuations any more, and a purely classical theory arises if these strings are used for 2nd quantization.

The zero $a_{2}^{\left(2 A^{-}\right)}=\frac{1}{2}$ could be interpreted as a dimensionless gravitational coupling strength, defined by $\alpha_{G}=\frac{1}{2}\left(E / m_{P l}\right)^{2}$, at the lightest black hole mass scale $E=m_{P l}$. Here $m_{P l}$ denotes the Planck mass. The backward

coupling form with $a_{2}^{\left(2 B^{-}\right)}=1$ would then describe two such black holes. There are no black holes lighter than of order of the Planck mass $m_{P l}$, since these immediately evaporate by Hawking radiation.

If this interpretation is correct, then the smallest zeros of the interaction energies $W(a)$ of the chaotic string theories fix the strengths of all four interactions. Electroweak interactions are fixed at the smallest fermionic mass scales $(N=3)$, strong interactions at the smallest bosonic mass scales $(N=2)$, gravitational interactions at the smallest black hole mass scales $(N=2, s=-1)$.

\section{Local minima of the self-energy $V(a)$}

\subsection{Self-interacting scalar field equations}

Let us now look at the other type of vacuum energy, the self-energy $V$ of the chaotic strings. As mentioned at the end of section 3, we expect that the self-energy is locally extremized for physically observable states.

One subset of free standard model parameters (the coupling strengths of the four interactions) was already fixed by the zeros of the interaction energy $W$, but for another subset of free parameters (essentially masses) the selfenergy $V$ may be the relevant quantity to look at. Consider an a priori free standard model coupling $\alpha$ that depends on such a free mass parameter. For example, $\alpha$ may be a Yukawa coupling. Again we assume that this standard 
model coupling $\alpha$ is a coupling $a$ in string space at the same time. We may regard $\alpha=a$ as a homogeneous self-interacting scalar field variable (similar to a dilaton field in superstring theory) with a complicated effective potential given by the expectation of the self energy $V(a)$ of the chaotic string. The stochastically quantized field equation reads

$$
\dot{a}=-\Gamma \frac{\partial V}{\partial a}+\text { noise }
$$

and if $\Gamma>0$ then local minima of the function $V(a)$ describe possible stable stationary states of standard model couplings. Any minimum of $V$ is thus a candidate for observed standard model couplings. We will denote the local minima of $V(a)$ as $a_{i}^{\prime(N b)}$.

Indeed a large number of local minima is observed that precisely coincide with known standard model interaction strengths. The reader not interested in all the details may directly proceed to section 6.6.

\subsection{The 3A string - weak and strong interactions of heavy fermion flavours}

Let us again start with the $3 \mathrm{~A}$ string. Fig. 9 shows the numerically deter-

mined function $V(a)$. First let us first look at rather large values of the coupling $a$. The minima labelled as

$$
\begin{aligned}
& a_{6}^{\prime(3 A)}=0.0953(1) \\
& a_{7}^{\prime(3 A)}=0.1677(5) \\
& a_{8}^{\prime(3 A)}=0.2327(5)
\end{aligned}
$$

seem to coincide with strong couplings at the heavy quark mass scales. A suitable Feynman web interpretation is given by $B_{1}$ massless, $B_{2}=g, f_{1}=q$, $\bar{f}_{2}=\bar{q}$, with $q=t, b, c$, respectively. Formula (13) with $N=3$ implies

$$
a_{i}^{\prime(3 A)}=\alpha_{s}\left(3 m_{q}\right) .
$$

The experimentally measured values of the free quark masses are $m_{c}=$ 1.26(3) $\mathrm{GeV}, m_{b}=4.22(4) \mathrm{GeV}, m_{t}=164(5) \mathrm{GeV}$ (these numerical values are obtained if one performs the average over the various results quoted in 
[9]). With the scale parameters $\Lambda^{\left(n_{f}\right)}$ determined earlier in section 5.5, the QCD formula in Appendix B yields the corresponding strong couplings as

$$
\begin{aligned}
& \alpha_{s}\left(3 m_{t}\right)=0.0952(3) \\
& \alpha_{s}\left(3 m_{b}\right)=0.1684(4) \\
& \alpha_{s}\left(3 m_{c}\right)=0.2323(20) .
\end{aligned}
$$

Apparently there is good coincidence with the observed local minima of the self energy of the 3A-string.

Further minima are observed that seem to describe weak interaction strengths of right-handed fermions. Remember that in the standard model the weak coupling constant is given by

$$
\alpha_{\text {weak }}=\alpha_{e l} \frac{\left(T_{3}-Q \sin ^{2} \theta_{W}\right)^{2}}{\sin ^{2} \theta_{W} \cos ^{2} \theta_{W}},
$$

where $Q$ is the electrical charge of the particle $(Q=-1$ for electrons, $Q=$ $2 / 3$ for $u$-like quarks, $Q=-1 / 3$ for $d$-like quarks), and $T_{3}$ is the third component of the isospin ( $T_{3}=0$ for right-handed particles, $T_{3}=-\frac{1}{2}$ for $e_{L}$ and $d_{L}, T_{3}=+\frac{1}{2}$ for $\nu_{L}$ and $\left.u_{L}\right)$. Consider right-handed fermions $f_{R}$. With $\sin ^{2} \theta_{W}=0.2318$ and the running electric coupling $\alpha_{e l}(E)$ taken at energy scale $E=3 m_{f}$ we obtain

$$
\begin{aligned}
& \alpha_{\text {weak }}^{d_{R}}\left(3 m_{d}\right)=0.000246 \\
& \alpha_{\text {weak }}^{c_{R}}\left(3 m_{c}\right)=0.001013 \\
& \alpha_{\text {weak }}^{e_{R}}\left(3 m_{e}\right)=0.00220 .
\end{aligned}
$$

On the other hand, we observe that $V(a)$ has minima at

$$
\begin{aligned}
& a_{1}^{\prime(3 A)}=0.000246(2) \\
& a_{2}^{\prime(3 A)}=0.00102(1) \\
& a_{3}^{\prime(3 A)}=0.00220(1)
\end{aligned}
$$

(see Fig. 9b. $a_{1}^{\prime}$ and $a_{3}^{\prime}$ are actually small local minima on top of the hill). There is good agreement with the weak coupling constants of $f_{R}=u_{R}, c_{R}, e_{R}$, respectively. A suitable Feynman web interpretation would be $B_{1}$ massless, $B_{2}=Z^{0}, f_{1}=f_{R}, \bar{f}_{2}=\bar{f}_{R}$. Basically, the minima yield statements on the charges of the particles involved. Generally, it is reasonable to assume that 
the above interaction states of $d, c$, and $e$ are not pure mass eigenstates but that small components of other flavours are mixed in as well. For example, $c$ could also have a small $t$ component, thus slightly increasing the relevant energy scale.

If $a_{1}^{\prime(3 A)}, a_{2}^{\prime(3 A)}, a_{3}^{\prime(3 A)}$ decribe right-handed fermions of type $d_{R}, u_{R}, e_{R}$ (mixed with higher flavors) then it is natural to assume that the next minimum $a_{4}^{\prime(3 A)}=0.00965(1)$ decribes a right-handed neutrino $\nu_{R}$. This could be a very heavy right-handed Majorana neutrino interacting gravitationally with $\alpha_{G}=$ $\frac{1}{2}\left(m_{\nu_{R}} / m_{P l}\right)^{2}=a_{4}^{\prime(3 A)}$. From this one obtains the mass $m_{\nu_{R}}=1.696(1) \cdot 10^{18}$ $\mathrm{GeV}$. We will come back to neutrinos in section 6.7.

The remaining minimum $a_{5}^{\prime(3 A)}=0.02145(3)$ inbetween weak minima $a_{1}^{\prime(3 A)}, a_{2}^{\prime(3 A)}, a_{3}^{\prime(3 A)}$ and strong minima $a_{6}^{\prime(3 A)}, a_{7}^{\prime(3 A)}, a_{8}^{\prime(3 A)}$ could decribe a unified coupling at the GUT scale. In fact, evolving the strong coupling $\alpha_{s}\left(3 m_{t}\right)=$ $a_{6}^{\prime(3 A)}$ to higher energies using the usual (non-supersymmetric) formulas [24, one obtains $\alpha_{s}\left(3 m_{Q}\right)=a_{5}^{\prime(3 A)}$ for $m_{Q} \approx 1.74 \cdot 10^{16} \mathrm{GeV}$, which is the GUT scale. At $E=m_{Q}$ the strong coupling $\alpha_{s}(E)$ and the weak coupling $\alpha_{2}(E)$ are observed to merge into the common value $\alpha_{s}=\alpha_{2}=0.02192$.

\subsection{The 3B string — further mixed states of heavy fermion flavours}

$V(a)$ for the $3 \mathrm{~B}$ string is plotted in Fig. 10. This string is of the backward coupling form, and our general arguments suggested that the backward coupling form describes mixed states of two particle species. We observe (among others) the following minima of the vacuum energy.

$$
\begin{aligned}
& a_{6}^{\prime(3 B)}=0.1027(1) \\
& a_{8}^{\prime(3 B)}=0.2916(5) .
\end{aligned}
$$

If we choose the same numerical values of the quark masses and the parameters $\Lambda^{\left(n_{f}\right)}$ as before, we obtain from the QCD-formula (111)

$$
\begin{aligned}
& \alpha_{s}\left(\frac{3}{2}\left(m_{t}+m_{b}\right)\right)=0.1027(4) \\
& \alpha_{s}\left(\frac{3}{2}\left(m_{c}+m_{s}\right)\right)=0.2914(35) .
\end{aligned}
$$

For the $s$-quark mass we used the value $m_{s}=(160 \pm 9) \mathrm{MeV}$ [23]. Again there is good coincidence between the above standard model couplings and the 
observed string couplings. The zeros $a_{6}^{\prime(3 B)}$ and $a_{8}^{\prime(3 B)}$ illustrate that up- and down quark flavours can mix flavour-independently for strong interaction.

At lower coupling strengths, the minimum $a_{3}^{\prime(3 A)}=0.00220$ of the $3 \mathrm{~A}$ string slightly changes to $a_{3}^{\prime(3 B)}=0.00223$ for the $3 \mathrm{~B}$ string. This numerically coincides with the weak coupling $\alpha_{\text {weak }}^{e_{R}}\left(\frac{3}{2}\left(m_{e}+m_{\mu}\right)\right)=0.00223$ of a state where $e$ and $\mu$ mix with equal weights. If this interpretation is correct, then the minimum $a_{3}^{\prime(3 B)}$ can be used to estimate the $\mu$ mass as being of the order of magnitude $100 \mathrm{MeV}$. Similarly, the minimum $a_{4}^{\prime(3 A)}$ describing the heavy right-handed Majorana neutrino changes by a small amount to $a_{4}^{\prime(3 B)}=0.00972(1)$, which could be due to neutrino flavor mixing.

Also the minima $a_{1}^{\prime(3 A)}$ and $a_{2}^{\prime(3 A)}$ of the $3 \mathrm{~A}$ string change by a very small positive amounts to $a_{1}^{\prime(3 B)}, a_{2}^{\prime(3 B)}$ for the $3 \mathrm{~B}$ string. It is difficult to numerically estimate this change, it is of the order of the precision by which we can determine the minima. It could again mean that small amounts of heavier flavours are mixing with $d, c$. For example, $d$ might get a small $s$ component and $c$ a small $t$ component. In total, one may conjecture that the four minima $a_{1}^{\prime(3 A)}, a_{2}^{\prime(3 A)}, a_{1}^{\prime(3 B)}, a_{2}^{\prime(3 B)}$ contain the information on the four mixing angles of the Kobayashi-Maskawa matrix.

At larger couplings $a$, the $N=3$ strings exhibit lots of further local minima. There is evidence that many of these can be associated with baryonic and mesonic states.

\subsection{The 2A string - further bosons}

Let us now turn to $N=2$ strings. The self-energy of the $2 \mathrm{~A}$ string theory is shown in Fig. 11. A very strongly pronounced minimum is

$$
a_{3}^{\prime(2 A)}=0.1848(1)
$$

It coincides with the strong coupling $\alpha_{s}$ at twice the $b$-quark mass. The Feynman web interpretation of this chaotic string state could be $B_{1}=B_{2}=$ $g, f_{1}=b, \bar{f}_{2}=\bar{b}$. Since $N=2$ the relevant energy scale is $E=(N / 2)\left(m_{g}+\right.$ $\left.2 m_{b}\right)=2 m_{b}$. Numerically, we get from eq. (111) for $m_{b}=4.23 \mathrm{GeV}$

$$
\alpha_{s}\left(2 m_{b}\right)=0.1848
$$

which coincides precisely with the minimum $a_{3}^{\prime(2 A)}$. In fact, the minimum is very sharp, moreover, it is in the region where the scale parameter $\Lambda^{(5)}$ is of 
relevance, which we know with high precision from section 5.5. Hence we may go the other way round and use $a_{3}^{\prime(2 A)}$ to predict $m_{b}$ with high precision. The value $\Lambda^{(5)}=0.20608(14) \mathrm{GeV}$ obtained earlier translates $a_{3}^{(2 A)}=0.1848(1)=$ $\alpha_{s}\left(2 m_{b}\right)$ to

$$
m_{b}=4.23(1) \mathrm{GeV} .
$$

This is in good agreement with estimates of the free $b$-mass using current algebra techniques [23].

Another minimum of interest is

$$
a_{2}^{\prime(2 A)}=0.03369(2)
$$

This seems to coincide with the weak coupling constant $\alpha_{2} \approx 1 / 30$ at the $Z^{0}$ mass scale. Our Feynman web interpretation is $B_{1}=Z^{0}, B_{2}=W_{\mu}^{0}$, $f_{1}=b, f_{2}=\bar{b}$, which implies

$$
a_{2}^{\prime(2 A)}=\alpha_{2}\left(m_{Z^{0}}+2 m_{b}\right)
$$

We can estimate the value of the weak coupling $\alpha_{2}$ at this energy scale using the well-known formulas for the running electroweak couplings [24]. At the $Z^{0}$ mass scale $m_{Z}=91.188 \mathrm{GeV}$ the QED formula (17) yields

$$
\alpha_{e l}\left(m_{Z}\right)=0.0078096
$$

The running electroweak mixing angle defined as $\hat{s}^{2}(E):=\alpha_{e l}(E) / \alpha_{2}(E)$ has been shown to very closely coincide with the effective mixing angle $\sin ^{2} \theta_{\text {eff }}^{\text {lept }}$ at the energy $E=m_{Z}$. One has [9]

$$
\hat{s}^{2}\left(m_{Z}\right)=\sin ^{2} \theta_{\text {eff }}^{\text {lept }}-0.00029
$$

Hence our result of $\sin ^{2} \theta_{\text {eff }}^{\text {lept }}=0.23177(7)$ derived in section 5.4 yields

$$
\hat{s}^{2}\left(m_{Z}\right)=0.23148(7)
$$

which implies

$$
\alpha_{2}\left(m_{Z}\right)=\frac{\alpha_{e l}\left(m_{Z}\right)}{\hat{s}^{2}}=0.03374(1) .
$$

If further transferring the running $\alpha_{2}$ to the slightly larger energy scale $m_{Z}+$ $2 m_{b}$ one obtains the numerical value

$$
\alpha_{2}\left(m_{Z}+2 m_{b}\right)=0.03369(1)
$$


which precisely coincides with the observed string coupling $a_{2}{ }^{\prime(2 A)}$ with a precision of 4 digits.

Finally, there is also a rather weakly pronounced minimum $a_{1}^{\prime(2 A)}=$ $0.00755(3)$ which could be interpreted as $\alpha_{e l}\left(2 m_{\tau}\right)$. In the Feynman web interpretation $B_{1}=B_{2}=\gamma, f_{1}=\tau, \bar{f}_{2}=\bar{\tau}$. This minimum could be used to estimate that the order of magnitude of the $\tau$ mass is about $2 \mathrm{GeV}$.

Summarizing, we see that whereas the interaction energy of the $2 \mathrm{~A}$ string provided evidence for the charged gauge bosons $W^{ \pm}$, the self energy of the 2A string provides evidence for the uncharged gauge bosons $g, Z^{0}$ and $\gamma$.

But clearly there are much more local minima of the self energy than the above three minima that we analyzed in detail. In fact, local minima of $V(a)$ accumulate near $a=0$ for all six chaotic string theories. It is unlikely that for all those minima simple standard model interpretations can be found. But we can always associate an observed minimum $a_{i}^{\prime}$ with a particle of mass $m_{i}$ that interacts by gravitational interaction with $\alpha_{G}=$ $\frac{1}{2}\left(m_{i} / m_{P l}\right)^{2}=a_{i}^{\prime}$. In this sense the many minima observed could simply mean that there are lots of particles that purely interact gravitationally but do not take part in standard model interactions. These could then be associated with dark matter. Chaotic strings seem to predict a very broad (though discrete) spectrum of dark matter particles, with masses covering all orders of magnitude.

\subsection{The 2B string - Yukawa interaction of the top quark}

Let us return to minima for which a standard model interpretation can be found. The self energy of the 2B string is shown in Fig. 12.

A very interesting minimum observed is

$$
a_{2}^{\prime(2 B)}=0.03440(2)
$$

This string coupling can be interpreted in terms of Yukawa interaction of the top quark. Our Feynman web interpretation is $B_{1}=B_{2}=H, f_{1}=t, f_{2}=\bar{t}$.

The Yukawa coupling of any fermion $f$ is proportional to the square of its mass. It is given by

$$
\alpha_{Y u}^{f}=\frac{1}{4} \alpha_{2} \cdot\left(\frac{m_{f}}{m_{W}}\right)^{2} .
$$


Still we have to decide on the energy scale $E$ for the running $\alpha_{2}(E)$. Our usual rules for the Feynman web interpretation $B_{1}=B_{2}=H, f_{1}=t, f_{2}=\bar{t}$ imply that the energy is given by $E=m_{H}+2 m_{t}$. Our interpretation of the minimum $a_{2}^{\prime(2 B)}$ is thus

$$
a_{2}^{\prime(2 B)}=\alpha_{Y u}^{t}\left(m_{H}+2 m_{t}\right)=\frac{1}{4} \alpha_{2}\left(m_{H}+2 m_{t}\right)\left(\frac{m_{t}}{m_{W}}\right)^{2} .
$$

Accepting this interpretation the formula allows for a very precise prediction of the top-mass $m_{t}$. From our earlier consideration we know that $\alpha_{2}\left(m_{Z}+\right.$ $\left.2 m_{b}\right)=0.03369(1)$. Transferring the running $\alpha_{2}$ to the higher energy scale $E=m_{H}+2 m_{t} \approx 2 m_{W}+2 m_{t}$ we obtain

$$
\alpha_{2}(E)=0.03284(1)
$$

(the usual (non-supersymmetric) formula for $\alpha_{2}(E)$ is used [24]). Solving for $m_{t}$ we get

$$
\frac{m_{t}}{m_{W}}=2 \sqrt{\frac{a_{2}^{\prime(2 B)}}{\alpha_{2}(E)}}=2.047(1)
$$

From the value $m_{W}=80.35(6) \mathrm{GeV}$ we thus get

$$
m_{t}=164.5(2) G e V \text {, }
$$

The corresponding pole mass $M_{t}$, of relevance for experiments, can be quite precisely determined by a formula in Degrassi et al. [18], which is based on $\Lambda^{(5)}$ and avoids $\Lambda^{(6)}$. We get

$$
M_{t}=174.4(3) \mathrm{GeV}
$$

The error only takes into account the precision by which we can determine $a_{2}^{\prime(2 B)}$, in addition there is the theoretical uncertainty whether our Feynman web interpretation of the minimum is correct. Nevertheless, our prediction coincides with the experimentally measured value $M_{t}=(174.3 \pm 5.1) \mathrm{GeV}$ [9] and is in fact more precise.

Let us estimate the largest possible systematic error of our prediction of the top-mass. The value of the Higgs mass is uncertain, for example it could turn out to be of the order $100 \mathrm{GeV}$ rather than our conjectured value of 154 $\mathrm{GeV}$. Also the error in $\alpha_{2}\left(m_{Z}\right)$ could be slightly underestimated. But all this would only mean that our top-mass prediction changes by less than $1 \mathrm{GeV}$. 


\subsection{Yukawa and gravitational interactions of all quarks and leptons}

Clearly not only the top-quark, but also the other heavy fermions are able to exhibit Yukawa interaction. The corresponding couplings are much smaller, since they all go with the mass squared of the particles involved. Nevertheless, they are indeed observed as suitable minima of the self-energy of the $2 \mathrm{~A} / 2 \mathrm{~B}$ string.

Generally, for all chaotic strings scaling behavior sets in if the coupling $a$ approaches 0 , and in this limit there is no difference between the forward and backward coupling form. One numerically observes for $a \rightarrow 0$

$$
V(a)-V(0)=f^{(N)}(\ln a) \cdot a^{\frac{1}{2}}
$$

where $f^{(N)}(\ln a)$ is a periodic function of $\ln a$ with period $\ln N^{2}$. Hence in a double logarithmic plot of $|V(a)-V(0)|$ versus $a$ one observes a straight line that is modulated by oscillating behaviour. From the periodicity it follows that if there is some local minimum at $a_{i}$ then there is also a minimum at $a_{i} / N^{2 n}$ for arbitrary $n$. Thus all local minima in the scaling region are only determined modulo $N^{2}$.

$\left|\log _{9}\right| V(a)-V(0)||$ is plotted for the 3A/B string in Fig. 13, and $\left|\log _{4}\right| V(a)-$ $V(0) \|$ for the $2 A / B$ string in Fig. 14. One has $V(0)=\frac{3}{8}$ for $N=3$ and $V(0)=0$ for $N=2$. Whereas the $3 \mathrm{~A} / \mathrm{B}$ string has only 2 minima per period (essentially describing the charge ratios of $d, u, e$, see section 6.2 ), the $2 \mathrm{~A} / \mathrm{B}$ string exhibits a much richer structure. For simplicity, let us denote the local minima of the $2 \mathrm{~A} / \mathrm{B}$ string in the scaling region as $b_{i}$. Within one period of length $\ln 4$, one observes 11 different minima (Fig. 15). These we have numerically determined in the region $[0.000143,0.000572]$ as

$$
\begin{aligned}
& b_{1}=0.000199(1) \\
& b_{2}=0.000263(2) \\
& b_{3}=0.000291(1) \\
& b_{4}=0.000306(1) \\
& b_{5}=0.000345(1) \\
& b_{6}=0.000368(3) \\
& b_{7}=0.000399(1) \\
& b_{8}=0.000469(1)
\end{aligned}
$$




$$
\begin{aligned}
b_{9} & =0.000482(1) \\
b_{10} & =0.000525(2) \\
b_{11} & =0.000558(1)
\end{aligned}
$$

(only $b_{i} \bmod 4$ is relevant). The remarkable fact is that these local minima of the self energy can be associated with Yukawa and gravitational couplings of all quark and lepton flavours modulo 4. Heavy particles turn out to minimize Yukawa couplingst light particles gravitational couplings. Leptons are found in the left part of Fig. 15, quarks in the right part (modulo 4).

Let us start with the heavy fermions $t, b, \tau, c$. The Yukawa coupling of the $t$ quark was already described by the minimum $a_{2}^{\prime(2 B)}=0.03440=: \tilde{b}_{5}$ outside the scaling region. This minimum can be formally regarded as evolving out of $b_{5}$ in the scaling region. The minima $b_{2}, b_{6}, b_{10}$ turn out to coincide with Yukawa couplings modulo 4 of $\tau, b, c$, respectively. We observe

$$
b_{i}=\alpha_{Y u}=\frac{1}{4} \alpha_{2}\left(m_{H}+2 m_{f}\right)\left(\frac{m_{f}}{m_{W}}\right)^{2} \cdot 4^{n},
$$

where $f$ denotes the fermion under consideration. Solving for $m_{f}$ we get a prediction of the heavy fermion mass modulo 2 .

$$
m_{f}=\sqrt{\frac{b_{i}}{\alpha_{2}\left(m_{H}+2 m_{f}\right)}} m_{W} 2^{-n+1}
$$

One obtains from the observed minima the following results.

\begin{tabular}{l|l|l|l|l|l}
$f$ & $\alpha_{2}\left(m_{H}+2 m_{f}\right)$ & $i$ & $b_{i} \cdot 10^{4}$ & $n$ & $m_{f}[\mathrm{GeV}]$ \\
\hline$t$ & 0.03284 & $(5)$ & $344.0(2)$ & 0 & $164.5(2)$ \\
$b$ & 0.03340 & 6 & $3.68(3)$ & 2 & $4.22(2)$ \\
$\tau$ & 0.03342 & 2 & $2.63(2)$ & 3 & $1.782(7)$ \\
$c$ & 0.03342 & 10 & $5.25(2)$ & 4 & $1.259(4)$
\end{tabular}

Note the small error in the mass predictions, which is due to the fact that Yukawa couplings are very sensitive to the fermion masses. On the other hand, the results are almost independent of the Higgs mass, respectively the precise value of $\alpha_{2}\left(m_{Z}\right)$. Of course, the integer $n$, which fixes the order of

\footnotetext{
${ }^{4}$ Note that we do observe the Yukawa couplings of the ordinary standard model and not those of a supersymmetric extension.
} 
magnitude of the predicted fermion masses, is not directly known from the scaling region of the $N=2$ string. However, the information on this integer is already given by other minima of other strings for larger couplings. For example, the order of magnitude of the $b$ mass follows from the minimum $a_{7}^{\prime(3 A)}$ or $a_{3}^{\prime(2 A)}$, that of the $c$ mass from the minimum $a_{8}^{\prime(3 A)}$ or $a_{2}^{\prime(3 A)}$, and that of the $\tau$ mass from the minimum $a_{1}^{\prime(2 A)}$. Hence $n$ is known from other string states. We may call $2^{n}$ a 'winding number' of the chaotic string.

Now let us proceed to the lighter fermions, and to the interpretation of the remaining minima $b_{i}$. Remarkably, for the light fermions where the mass is known with rather high precision we observe that the self energy has local minima for couplings that coincide with gravitational couplings modulo 4 . We observe for $i=1,4,7,8,9$

$$
b_{i}=\alpha_{G}=\frac{1}{2}\left(\frac{m_{f}}{m_{P l}}\right)^{2} \cdot 4^{n},
$$

or, solving for $m_{f}$, we obtain the prediction

$$
m_{f}=\sqrt{2 b_{i}} m_{P l} 2^{-n}
$$

of the light fermion masses. The result is shown in the following table.

\begin{tabular}{l|l|l|l|l}
$f$ & $i$ & $b_{i} \cdot 10^{4}$ & $n$ & $m_{f}[\mathrm{MeV}]$ \\
\hline$\mu$ & 1 & $1.99(1)$ & 61 & $105.6(3)$ \\
$\mathrm{e}$ & 4 & $3.06(1)$ & 69 & $0.5117(8)$ \\
$d$ & 7 & $3.99(1)$ & 65 & $9.35(1)$ \\
$u$ & 8 & $4.69(1)$ & 66 & $5.07(1)$ \\
$s$ & 9 & $4.82(1)$ & 61 & $164.4(2)$
\end{tabular}

Again the winding number $2^{n}$ determining the order of magnitude of the mass is not known from the scaling region but already fixed by other string states. For example, the zeros $a_{2}^{(3 A)}, a_{1}^{(3 A)}, a_{1}^{(3 B)}$ of the interaction energy $W(a)$ yield the order of magnitude of the masses of $e, d, u$, respectively. The order of magnitude of the $\mu$ mass follows from $a_{3}^{\prime(3 B)}$, and that of the $s$ mass from $a_{8}^{\prime(3 B)}$.

\footnotetext{
${ }^{5}$ Averaging the various results on light quark masses in the particle data listing [9], one obtains the values $m_{u}=4.7(9) \mathrm{MeV}, m_{d}=9.1(6) \mathrm{MeV}, m_{s}=167(7) \mathrm{MeV}$ at the proton mass renormalization scale.
} 
Generally, it is a very fortunate effect that scaling behaviour of the vacuum energy sets in for small $a$. For example, we would never be able to get any information on the gravitational coupling of an electron $\alpha_{G}\left(m_{e}\right)=$ $\frac{1}{2}\left(m_{e} / m_{P l}\right)^{2}=8.76 \cdot 10^{-45}$ in a direct simulation with $a=\alpha_{G}$. However, we can easily iterate the coupled map lattice with the much larger coupling $a=\alpha_{G}\left(m_{e}\right) \cdot 4^{69}=0.000305$ and then conclude onto $\alpha_{G}\left(m_{e}\right)$ modulo 4 via the scaling argument.

It is straightforward to conjecture that the remaining minima can be associated with massive neutrino states. This will be worked out in detail in the next section.

\subsection{Neutrino mass prediction}

It is desirable to find an interpretation of all minima that possesses the highest symmetry standards. It is in fact possible to provide a fully symmetric attribution of the 11 minima to the 12 known fermions if one assumes that the minimum $b_{1}$ is degenerated, i.e. that it describes two different particles with the same mass modulo 2 . This scheme of largest symmetry is described by the following table.

\begin{tabular}{c|c|c|c|c|c|c|c|c|c|c|c}
$b_{0}$ & $b_{1}^{(1)}$ & $b_{1}^{(2)}$ & $b_{2}$ & $b_{3}$ & $b_{4}$ & $b_{5}$ & $b_{6}$ & $b_{7}$ & $b_{8}$ & $b_{9}$ & $b_{10}$ \\
\hline$\nu_{2}$ & $\mu$ & $\nu_{3}$ & $\tau$ & $\nu_{1}$ & $e$ & $(t)$ & $b$ & $d$ & $u$ & $s$ & $c$
\end{tabular}

Since all minima are only defined modulo 4 , we have identified $b_{0}:=b_{11} / 4$. The $t$ quark is put into parenthesis since $m_{t}$ is so large that the corresponding Yukawa coupling falls out of the scaling region. Within the above scheme all up and down members of the same family are described by neighbored minima, and the up member always has a larger self energy than the down member (see Fig. 15). All leptons are grouped together (in family index order $2,3,1$ ) and all quarks as well (in family index order $3,1,2$ ). The fact that for the $N=2$ string one minimum is degenerated is completely analogous and symmetric to the $N=3$ string, where also one minimum was degenerated, describing the fact that electrons and $d$-quarks have the same charge modulo 3 (see section 6.2).

The above scheme associates the three neutrino states $\nu_{1}, \nu_{2}, \nu_{3}$ with the

minima $b_{3}, b_{0}, b_{1}^{(2)}$, respectively, and hence these minima fix the neutrino 
masses modulo 2 as

$$
\begin{aligned}
& m_{\nu_{1}}=\sqrt{2 b_{3}} m_{P l} 2^{-n_{1}} \\
& m_{\nu_{2}}=\sqrt{2 b_{0}} m_{P l} 2^{-n_{2}} \\
& m_{\nu_{3}}=\sqrt{2 b_{1}^{(2)}} m_{P l} 2^{-n_{3}}
\end{aligned}
$$

or equivalently

$$
\begin{aligned}
& m_{\nu_{1}}=0.952(1) \mathrm{eV} \bmod 2 \\
& m_{\nu_{2}}=1.318(1) \mathrm{eV} \bmod 2 \\
& m_{\nu_{3}}=1.574(4) \mathrm{eV} \bmod 2 .
\end{aligned}
$$

Since the relevant string coupling constant is the gravitational coupling $\alpha_{G}=$ $\frac{1}{2}\left(m_{\nu} / m_{P l}\right)^{2}$ one expects that these values represent the masses of the mass eigenstates $\nu_{1}, \nu_{2}, \nu_{3}$ (rather than those of the weak eigenstates $\nu_{e}, \nu_{\mu}, \nu_{\tau}$ ).

To obtain concrete numerical values we have to decide on the relevant powers of 2. Let us here be guided by symmetry considerations with the other fermions. Define the open intervals $I_{1}$ and $I_{2}$ as

$$
I_{1}=\left(2^{4}, 2^{5}\right) \quad I_{2}=\left(2^{7}, 2^{8}\right)
$$

We observe

$$
\begin{aligned}
& \frac{m_{t}}{m_{c}}=130.7 \in I_{2} \\
& \frac{m_{c}}{m_{u}}=248.3 \in I_{2} \\
& \frac{m_{b}}{m_{s}}=25.67 \in I_{1} \\
& \frac{m_{s}}{m_{d}}=17.58 \in I_{1} \\
& \frac{m_{\tau}}{m_{\mu}}=16.83 \in I_{1} \\
& \frac{m_{\mu}}{m_{e}}=206.7 \in I_{2},
\end{aligned}
$$

i.e. all up-type quark mass ratios are in $I_{2}$, all down-type quark mass ratios in $I_{1}, 3 \mathrm{rd} / 2 \mathrm{nd}$ family lepton mass ratios are in $I_{1}, 2 \mathrm{nd} / 1$ st family lepton mass 
ratios in $I_{2}$. The most reasonable assumption giving full symmetry to the problem is to assume that neutrinos follow this general pattern as well, i.e.

$$
\begin{aligned}
& \frac{m_{3}}{m_{2}} \in I_{1} \\
& \frac{m_{2}}{m_{1}} \in I_{2}
\end{aligned}
$$

So far the most stringent experimental evidence for neutrino masses comes from atmospheric neutrinos, providing evidence for

$$
\Delta m_{a}^{2} \approx 3 \cdot 10^{-3} e V^{2}
$$

and maximal mixing [9]. If there is a hierarchy of neutrino masses $m_{\nu_{3}}>>$ $m_{\nu_{2}}>m_{\nu_{1}}$ then the experimental result can be interpreted as $m_{\nu_{3}} \approx$ $\sqrt{\Delta m_{a}^{2}} \sim 0.055 \mathrm{eV}$. If this experimental estimate is correct with a precision of a factor 2, then eq. (78) together with (91) implies $n_{3}=92$, and eqs. (77), (76) together with (89), (90) imply $n_{2}=96, n_{1}=104$. This means that the chaotic string spectrum yields the very precise predictions

$$
\begin{aligned}
& m_{\nu_{1}}=1.452(3) \cdot 10^{-5} \mathrm{eV} \\
& m_{\nu_{2}}=2.574(3) \cdot 10^{-3} \mathrm{eV} \\
& m_{\nu_{3}}=4.92(1) \cdot 10^{-2} \mathrm{eV} .
\end{aligned}
$$

We may also try to avoid the experimental input (91) and use a purely theoretical argument to fix the relevant energy scale. As described in section 6.2 , the minimum $a_{4}^{\prime(3 A)}$ can be theoretically interpreted as indicating the existence of a very heavy right-handed neutrino $\nu_{R}$ of mass $m_{\nu_{R}}=1.696(1) \cdot 10^{18}$ $\mathrm{GeV}$. The seesaw mechanism [25, 26] provides an estimate of the light (lefthanded) neutrino masses from the heavy right-handed ones via the equation

$$
m_{\nu_{L}} \approx \frac{m_{q}^{2}}{m_{\nu_{R}}}
$$

where $m_{q}$ is of the order of magnitude of a typical quark mass. Choosing $m_{q}=m_{t}$ eq. (95) yields $m_{\nu_{L}} \approx 1.59 \cdot 10^{-5} \mathrm{eV}$. This is very close to the value of $m_{\nu_{1}}$ in eq. (92), thus suggesting that $\nu_{L}$ is an electron neutrino and that there is almost no mixing of $\nu_{1}$ with $\nu_{2}\left(\right.$ or $\left.\nu_{3}\right)$ to form $\nu_{e}$, i.e. $\nu_{e} \approx \nu_{1}$. In this way one again ends up with $n_{1}=104$ and eqs. (92)-(94) are obtained by purely theoretical arguments. The predicted value for $m_{\nu_{2}}$ is consistent 
with present experimental measurements, i.e. the low-mixing angle (LMA) solution of the solar neutrino problem. The experiments provide evidence for $\Delta m_{s}^{2} \approx 5.4 \cdot 10^{-6} \mathrm{eV}^{2}$ [9], which could be interpreted as $m_{\nu_{2}} \approx 2.3 \cdot 10^{-3} \mathrm{eV}$, in very good agreement with eq. (93).

\subsection{The $2 A^{-}$and $2 B^{-}$strings - mass ratio of heavy bosons}

The fermion mass ratios were determined by the minima $b_{i}$ of the $2 \mathrm{~A} / 2 \mathrm{~B}$ string, according to the relation

$$
\frac{b_{i_{1}}}{b_{i_{2}}}=\left(\frac{m_{f_{1}}}{m_{f_{2}}}\right)^{2} \bmod 4
$$

The quadratic dependence comes from the quadradic energy dependence of gravitational (or Yukawa) couplings.

It now seems plausible that also boson mass ratios could be fixed in a similar way, i.e. by the self energy of some suitable string theories in the limit of very small $a$. The most plausible remaining candidates are the $2 \mathrm{~A}^{-} / 2 \mathrm{~B}^{-}$ strings. The self energy of these strings also exhibits periodic scaling behaviour with period ln 4 for $a \rightarrow 0$, but for the anti-diffusive coupling form only two minima per period are observed, which we have determined in the region $[0.000143,0.000572]$ as $b_{1}^{-}=0.000335(1)$ and $b_{2}^{-}=0.000361(2)$ (Fig. 16). In analogy to the fermionic case we may assume that the bosonic mass ratios are given by

$$
\frac{b_{1}^{-}}{b_{2}^{-}}=\left(\frac{m_{B_{1}}}{m_{B_{2}}}\right)^{2} \bmod 4 .
$$

Suppose one of the minima describes the $W$-boson, of mass $80.35(6) \mathrm{GeV}$, and the other one the Higgs boson. Then, depending on which minimum is identified with which particle as well as the unknown power of 4 , the above equation allows for the following masses of the Higgs boson: 77.4, 83.4, 154.8, 166.8, 309.6, 333.6 GeV. But experimental and theoretical bounds [9] imply $95 \mathrm{GeV}<m_{H}<190 \mathrm{GeV}$, hence only the values $154.8(7)$ or $166.8(7) \mathrm{GeV}$ survive. It is remarkable that (within the error bars) the value $154.8(7) \mathrm{GeV}$ coincides with the value $154.4(4) \mathrm{GeV}$ that was predicted independently in section 5.7. This value implies that the minimum $b_{1}^{-}$is attributed to $W$ and 
$b_{2}^{-}$to $H$. Numerically, one observes the relation

$$
b_{i}^{-}=\frac{1}{2} a_{4}^{\prime(3 A)}\left(\frac{m_{P l}}{m_{B_{i}}}\right)^{2} \bmod 4=\left(\frac{m_{\nu_{R}}}{m_{B_{i}}}\right)^{2} \bmod 4,
$$

which suggests that what is really fixed by the $2 A^{-} / 2 B^{-}$string is the ratio $m_{\nu_{R}} / m_{B_{i}}$ between fermion and boson masses. Note that the coupling $b_{i}^{-}$is proportional to an inverse gravitational coupling. This reminds us of the concept of duality in superstring theories, where couplings are replaced by inverse couplings.

\section{Summary and Outlook}

Instead of considering the standard model alone and putting in about 25 free parameters by hand, in this paper we have suggested to postulate the existence of chaotic strings underlying the noise of the Parisi-Wu approach of stochastic quantization on a very small scale. The chaotic string dynamics can be physically interpreted as a 1-dimensional strongly fluctuating dynamics of vacuum fluctuations. It generates effective potentials which distinguish the observed standard model couplings from arbitrary ones. The dynamics may either have determined the standard model parameters at a very early stage of the universe (e.g. in a pre-big bang scenario) or it may still evolve today and stabilize the observed values of the parameters.

Whereas for standard model fields, as well as for superstrings after compactification, continuous gauge symmetries such as $U(1), S U(2)$ or $S U(3)$ are relevant, for the chaotic strings a discrete $Z_{2}$ symmetry is relevant. Whereas standard model fields or ordinary strings usually evolve in a regular way, the chaotic strings obtained for $N>1$ evolve in a deterministic chaotic way 0 . They are strongly self-interacting and correspond to a Bernoulli shift of information for vanishing spatial coupling $a$. The constraint conditions on the vacuum energy (or the analogues of the Einstein and scalar field equations) fix certain equilibrium metrics in string space, which determine the strength of the Laplacian coupling. We have provided extensive numerical evidence that these equilibrium metrics reproduce the free standard model parameters with very high precision. Essentially coupling constants are fixed by the interaction energy $W(a)$, and masses, mass mixing angles and charges by the self energy $V(a)$. This is summarized in Fig. 17.

\footnotetext{
${ }^{6}$ For $N=1$ there is no chaotic behavior and one just obtains a discretized heat equation.
} 
The simplest physical interpretation is to regard the chaotic string dynamics as a dynamics of vacuum fluctuations, which is present everywhere but which is unobservable due to the uncertainty relation. Only expectations of the dynamics can be measured, in terms of the fundamental constants of nature. The dynamics may have fixed the free parameters already at a very early (pre-big bang) stage of the universe.

On the other hand, one may also wish to embedd chaotic strings into more general theories, for example M-theory. Several different approaches seem possible. One might try to attribute to each of the 6 components that make up M-theory in moduli space one of the 6 chaotic string theories, used for second quantization. A possible picture is plotted in Fig. 18. Many other scenarios seem possible, and it is clear that at the present stage any diagram of the type of Fig. 18 is merely speculation. What, however, is clear is that an ordinary string winding around a compactified space has a discrete momentum spectrum, and the string field variable $X^{\mu}(\sigma, \tau)$ is a kind of position variable taking on continuous values. On the contrary, a chaotic string has a discrete position spectrum $i$ and the field variable $\Phi_{n}^{i}$ is a kind of momentum variable taking on continuous values. Hence in that sense the role of position and momentum is exchanged.

An interesting possibility is that after compactification each of the 6 chaotic strings might wind around one of the 6 compactified dimensions, respecting the relevant (unknown) topological structure of the compactified space. Note that if chaotic strings live in the compactified space then they do not 'disturb' our usual understanding of 4-dimensional space-time physics. Rather, they yield a very relevant amendment. Each inverse string coupling constant $a^{-1}$ can be regarded as an element of the metric in the compactified space, and the analogues of the Einstein- and scalar field equations make the observed standard model parameters evolve to the minima of the effective potentials. This would give physical meaning to the compactified space, in the sense that at each ordinary space-time point the standard model parameters are known and in fact stabilized, due to the chaotic dynamics in the compactified space.

Generally, the chaotic strings exhibit symmetry under the replacement $T_{N} \rightarrow-T_{N}$, which could be formally associated with a supersymmetry transformation. However, when introducing the evolution equations (15) and (35) of the couplings one has to decide on the sign of the constant $\Gamma$. This choice effectively breaks supersymmetry. Generally, it seems reasonable to assume that with such a choice of sign the expectation of the vacuum energy of the 
chaotic noise strings effectively breaks supersymmetry and singles out the physically relevant string vacua of superstring theory. Supersymmetric partners of ordinary particles might be formally described by maxima rather than minima of the effective potentials of the chaotic strings — but these string states are unstable states in fictitious time. The instability might indicate that supersymmetric partners, though formally there to cancel divergences in the Feynman diagrams as well as unwanted vacuum energy, may turn out to be unobservable in our world.

\section{Appendix A: large coupling limit of a $\phi^{4}-$ theory}

Consider a $\phi^{4}$-theory in 1 dimension that is stochastically quantized. The field equation is

$$
\frac{\partial}{\partial t} \phi(x, t)=\left(\frac{\partial^{2}}{\partial x^{2}}-\mu^{2}\right) \phi(x, t)-\lambda \phi^{3}(x, t)+\text { noise. }
$$

Discretizing space $x$ with lattice constant $\delta$ and fictitious time $t$ with lattice constant $\tau$ one obtains

$$
\frac{\Phi_{n+1}^{i}-\Phi_{n}^{i}}{\tau}=\frac{\Phi_{n}^{i+1}-2 \Phi_{n}^{i}+\Phi_{n}^{i-1}}{\delta^{2}}-\mu^{2} \Phi_{n}^{i}-\lambda \Phi_{n}^{i 3}+\text { noise. }
$$

This can be written as

$$
\Phi_{n+1}^{i}=\left(1-2 \frac{\tau}{\delta^{2}}-\mu^{2} \tau\right) \Phi_{n}^{i}-\lambda \tau \Phi_{n}^{i^{3}}+\frac{\tau}{\delta^{2}}\left(\Phi_{n}^{i+1}+\Phi_{n}^{i-1}\right)+\tau \cdot \text { noise }
$$

Now let $\tau \rightarrow 0, \delta \rightarrow 0,-\mu^{2} \rightarrow \infty, \lambda \rightarrow \infty$ such that

$$
\begin{array}{rll}
\frac{\tau}{\delta^{2}} & =: \frac{a}{2} \text { finite } \\
\mu^{2} \tau & =: \mu_{\text {ren }}^{2} & \text { finite } \\
\lambda \tau & =: \lambda_{\text {ren }} & \text { finite. }
\end{array}
$$

Eq. (101) then reduces to a coupled map lattice of the form

$$
\Phi_{n+1}^{i}=(1-a) T\left(\Phi_{n}^{i}\right)+\frac{a}{2}\left(\Phi_{n}^{i+1}+\Phi_{n}^{i-1}\right) .
$$


The local map $T$ is given by

$$
T(\Phi)=\left(1-\frac{\mu_{r e n}^{2}}{1-a}\right) \Phi-\frac{\lambda_{r e n}}{1-a} \Phi^{3} .
$$

The noise term $\tau \cdot$ noise actually vanishes in the limit $\tau \rightarrow 0$ that is considered here. A local map with strongest possible chaotic behaviour is obtained for the choice

$$
\begin{aligned}
& \mu_{\text {ren }}^{2}=-2(1-a) \\
& \lambda_{\text {ren }}=4(1-a)
\end{aligned}
$$

This yields the negative third-order Tschebyscheff polynomial $-T_{3}(\Phi)=$ $3 \Phi-4 \Phi^{3}$. Equally well we can also obtain the positive Tchebyscheff polynomial $T_{3}(\Phi)$ for the choice

$$
\begin{aligned}
& \mu_{r e n}^{2}=4(1-a) \\
& \lambda_{\text {ren }}=-4(1-a)
\end{aligned}
$$

The result is the chaotic $3 B$ string $(N=3, b=0, s=1)$.

We could also start from a $\phi^{3}$-theory, getting in a similar way an $N=2$ string. Moreover, instead of doing the nearest-neighbour coupling with $\Phi_{n}^{i-1}$ and $\Phi_{n}^{i+1}$, we could equally well choose the updated variables $T\left(\Phi_{n}^{i-1}\right)$ and $T\left(\Phi_{n}^{i+1}\right)$. This yields a chaotic strings with $b=1$ rather than $b=0$. Finally, replacing $T$ by $-T$ at odd lattice sites yields the anti-diffusive coupling form $s=-1$.

\section{Appendix B: QCD formulas used}

In a third-order QCD calculation the running strong coupling is given by

$$
\begin{aligned}
\alpha_{s}(E) & =\frac{-2}{b_{0} \ln \frac{E^{2}}{\Lambda^{2}}}\left\{1+\frac{2 b_{1} \ln \ln \frac{E^{2}}{\Lambda^{2}}}{b_{0}^{2} \ln \frac{E^{2}}{\Lambda^{2}}}\right. \\
& \left.+\frac{4 b_{1}^{2}}{b_{0}^{4}\left(\ln \frac{E^{2}}{\Lambda^{2}}\right)^{2}}\left[\left(\ln \ln \frac{E^{2}}{\Lambda^{2}}-\frac{1}{2}\right)^{2}+\frac{b_{0} b_{2}}{b_{1}^{2}}-\frac{5}{4}\right]\right\} \\
& +O\left(\frac{1}{\left(\ln \frac{E^{2}}{\Lambda^{2}}\right)^{4}}\right) .
\end{aligned}
$$


Here $\Lambda$ is the QCD scale parameter, which takes on different values in the various flavour regions, and the coefficients $b_{i}$ are given by

$$
\begin{aligned}
& b_{0}=-\frac{1}{2 \pi}\left(11-\frac{2 n_{f}}{3}\right) \\
& b_{1}=-\frac{1}{4 \pi^{2}}\left(51-\frac{19 n_{f}}{3}\right) \\
& b_{2}=-\frac{1}{64 \pi^{3}}\left(2857-\frac{5033 n_{f}}{9}+\frac{325 n_{f}^{2}}{27}\right) .
\end{aligned}
$$

$n_{f}$ denotes the relevant number of quark flavours. The integer $n_{f}$ changes by 1 at thresholds given by the quark pole masses $M_{q}$. At the thresholds $M_{q}$, $\alpha_{s}(E)$ should be continuous. This determines the scale parameters $\Lambda^{\left(n_{f}\right)}$ in the various flavour regions, given one of the parameters $\Lambda^{\left(n_{f}\right)}$ for some $n_{f}$ (or, equivalently, given $\alpha_{s}\left(E^{*}\right)$ at some fixed energy $\left.E^{*}\right)$. The relation between pole quark masses $M_{q}$ and free quark masses $m_{q}$ is

$$
M_{q}=m_{q}\left\{1+\frac{4}{3} \frac{\alpha_{s}\left(M_{q}\right)}{\pi}+K_{q}\left(\frac{\alpha_{s}\left(M_{q}\right)}{\pi}\right)^{2}+O\left(\left(\frac{\alpha_{s}\left(M_{q}\right)}{\pi}\right)^{3}\right)\right\},
$$

with

$$
K_{q}=16.11-1.04 \sum_{i=1}^{n_{f}-1}\left(1-\frac{M_{i}}{M_{q}}\right)
$$

22]. By free quark mass we actually mean the $\overline{M S}$ running quark mass $\bar{m}_{q}(E)$ at renormalization scale $E=M_{q}$. For the light quarks $u, d, s$ we define the free masses to be the running $\overline{M S}$ masses at a renormalization scale given by the proton energy scale $E \approx 1 \mathrm{GeV}$.

\section{References}

[1] MB Green, JH Schwarz, E Witten, Supersting Theory, Vol I and II, Cambridge University Press, Cambridge (1987)

[2] J Polchinski, String Theory, Vol I and II, Cambridge University Press, Cambridge (1998)

[3] JP Gauntlett, M-Theory: Strings, Duality and Branes, QMW-PH-98-2 
[4] G Parisi, YS Wu, Sci. Sin. 24, 483 (1981)

[5] PH Damgaard, H Hüffel, eds., Stochastic Quantization, Word Scientific, Singapore (1988)

[6] K. Kaneko (ed.), Theory and Applications of coupled map lattices, Wiley, New York (1993)

[7] C Beck, Nonlinearity 8, 423 (1995)

[8] A Hilgers, C Beck, Phys. Rev. 60E, 5385 (1999)

[9] Particle Data Group, DE Groom et al., Eur. Phys. J. C15, 1 (2000), http://pdg.lbl.gov

[10] C Beck, Nonlinearity 4, 1131 (1991)

[11] P Billingsley, Convergence of probability measures, Wiley, New York (1968)

[12] C Beck, G Roepstorff, Physica 145A, 1 (1987)

[13] A Hilgers, C Beck, Higher-order correlations of Tchebyscheff maps, to appear in Physica D (2001)

[14] C Beck, Phys. Lett. 248A, 386 (1998)

[15] C Beck, F Schlögl, Thermodynamics of Chaotic Systems, Cambridge University Press, Cambridge (1993)

[16] C Tsallis, J. Stat. Phys. 52, 479 (1988)

[17] JG Gilson, Phys. Essays 9, 342 (1996)

[18] G Degrassi, P Gambino, A Sirlin, Phys. Lett. 394B, 188 (1997)

[19] WJ Marciano, Phys. Rev. 29D, 580 (1984)

[20] M Boglione, MR Pennington, Phys. Rev. Lett. 97, 1998 (1997)

[21] KJ Peach, LLJ Vick, eds., High energy phenomenology, Proc. 42nd Scottish Universities Summer School in Physics, St. Andrews, IOP Publishing, London (1994)

[22] N Gray, DJ Broadhurst, W Grafe, K Schilcher, Zeitschr. Phys. 48C, 673 (1990)

[23] S Narison, Phys. Lett. 341B, 73 (1994)

[24] U Amaldi, W de Boer, H Fürstenau, Phys. Lett. 260B, 447 (1991)

[25] M Gell-Mann, P Ramond, R Slansky, in Supergravity, eds. P van Nieuwenhuizen and D Freedman, North Holland, Amsterdam (1979)

[26] D Falcone, F Tramontano, Phys. Rev. 63D, 073007 (2001) 


\section{Figure captions}

Fig. 1 Feynman web interpretation of the chaotic string dynamics.

Fig. 2 Evolution of standard model coupling parameters to stable zeros $a_{1}, a_{2}$ of the interaction energy.

Fig. 3 Interaction energy $W(a)$ of the chaotic $3 A$ string in the region $a \in$ $[0,1]$ (a) and $a \in[0,0.018](\mathrm{b}) . W(a)$ is numerically obtained by iterating eq. (11) with $N=3$ and $b=1$ for random initial conditions and averaging $\frac{1}{2} \Phi_{n}^{i} \Phi_{n}^{i+1}$ over all $n$ and $i$.

Fig. 4 Interaction energy $W(a)$ of the chaotic $3 B$ string in the region $a \in$ $[0,1]$ (a) and $a \in[0,0.018]$ (b).

Fig. 5 Interaction energy $W(a)$ of the chaotic $2 A$ string.

Fig. 6 Interaction energy $W(a)$ of the chaotic $2 B$ string.

Fig. 7 Interaction energy $W(a)$ of the chaotic $2 A^{-}$string.

Fig. 8 Interaction energy $W(a)$ of the chaotic $2 B^{-}$string.

Fig. 9 Self energy $V(a)$ of the chaotic $3 A$ string in the region $a \in[0,1]$ (a) and $a \in[0,0.022]$ (b). $V(a)$ is numerically obtained by iterating eq. (11) with $N=3$ and $b=1$ for random initial conditions and averaging $\frac{3}{2}\left(\Phi_{n}^{i}\right)^{2}-\left(\Phi_{n}^{i}\right)^{4}$ over all $n$ and $i$.

Fig. 10 Self energy $V(a)$ of the chaotic $3 B$ string.

Fig. 11 Self energy $V(a)$ of the chaotic $2 A$ string. $V(a)$ is obtained by iterating eq. (1) with $N=2$ and $b=1$ and averaging $\Phi_{n}^{i}-\frac{2}{3}\left(\Phi_{n}^{i}\right)^{3}$ over all $n$ and $i$.

Fig. 12 Self energy $V(a)$ of the chaotic $2 B$ string.

Fig. $13|\log | V(a)-V(0)||$ versus $\log a$ for the $3 A / B$ string in the scaling region.

Fig. $14|\log | V(a)-V(0)||$ versus $\log a$ for the $2 A / B$ string in the scaling region.

Fig. 15 One period of the self energy of the $2 A / 2 B$ string in the scaling region. The various minima fix the fermion masses.

Fig. 16 Same as Fig. 15, but for the $2 A^{-} / 2 B^{-}$string. The minima fix the boson masses. 
Fig. 17 Summary of the way in which the 2 types of vacuum energies of the 6 types of chaotic string theories fix standard model parameters, (a) interaction energy $W(a)$, (b) self energy $V(a)$.

Fig. 18 A possible correspondence between superstrings and chaotic strings. The left hand side shows the 5 known superstring theories plus the 11-dimensional theory, which compactified on a circle is dual to the IIA string and compactified on an interval dual to the HET E8 $\times E 8$ string. The right hand side shows a similar diagram for the chaotic string theories. 


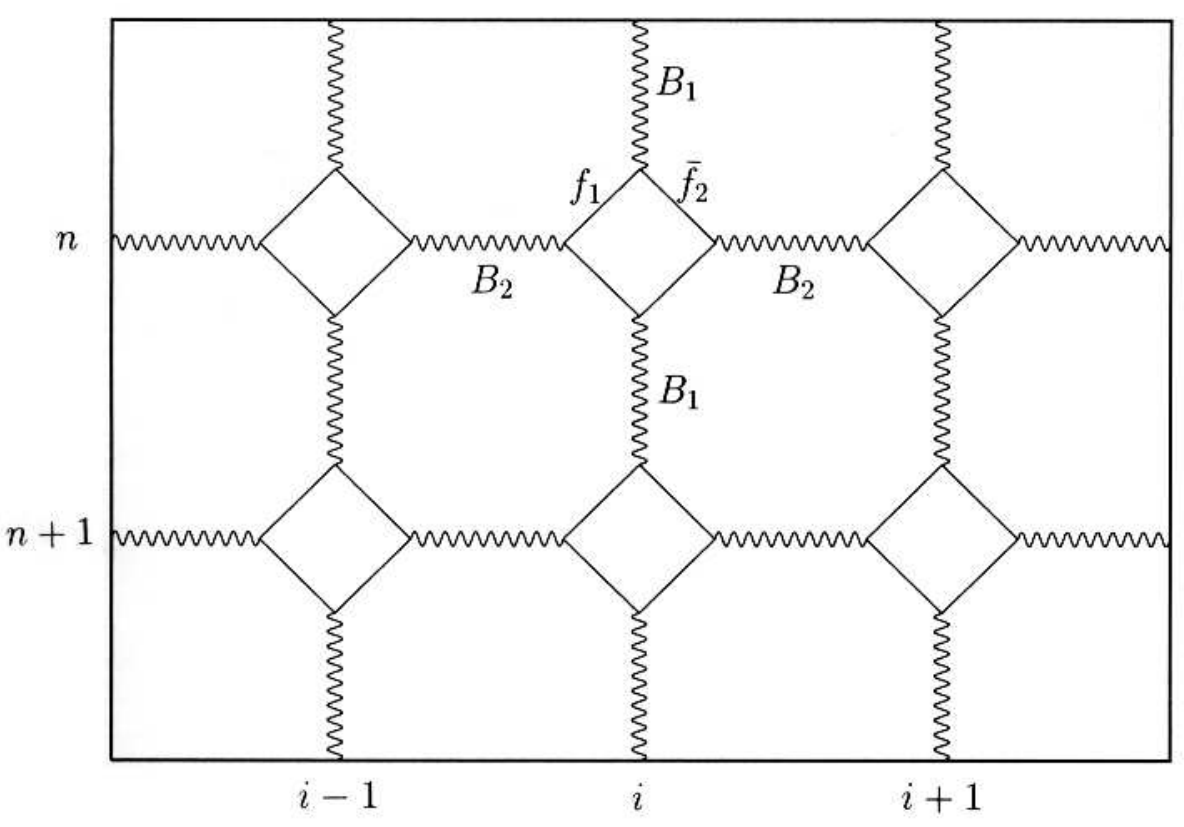

Fig. 1 


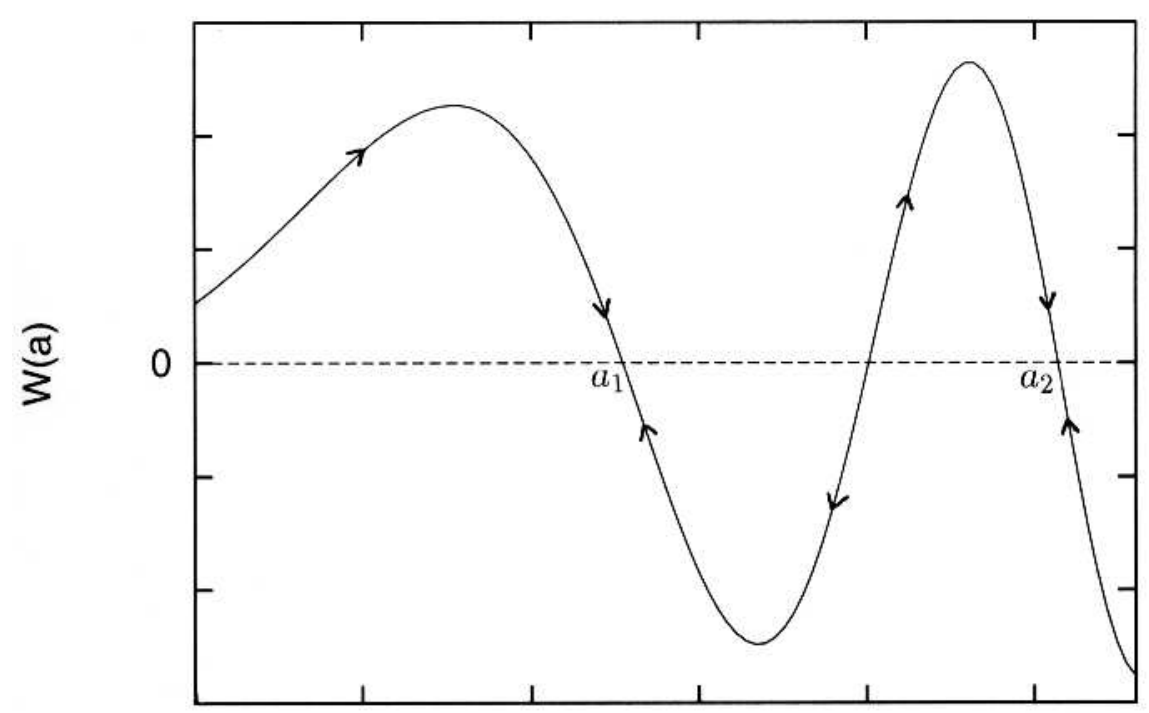

Fig. 2

a 


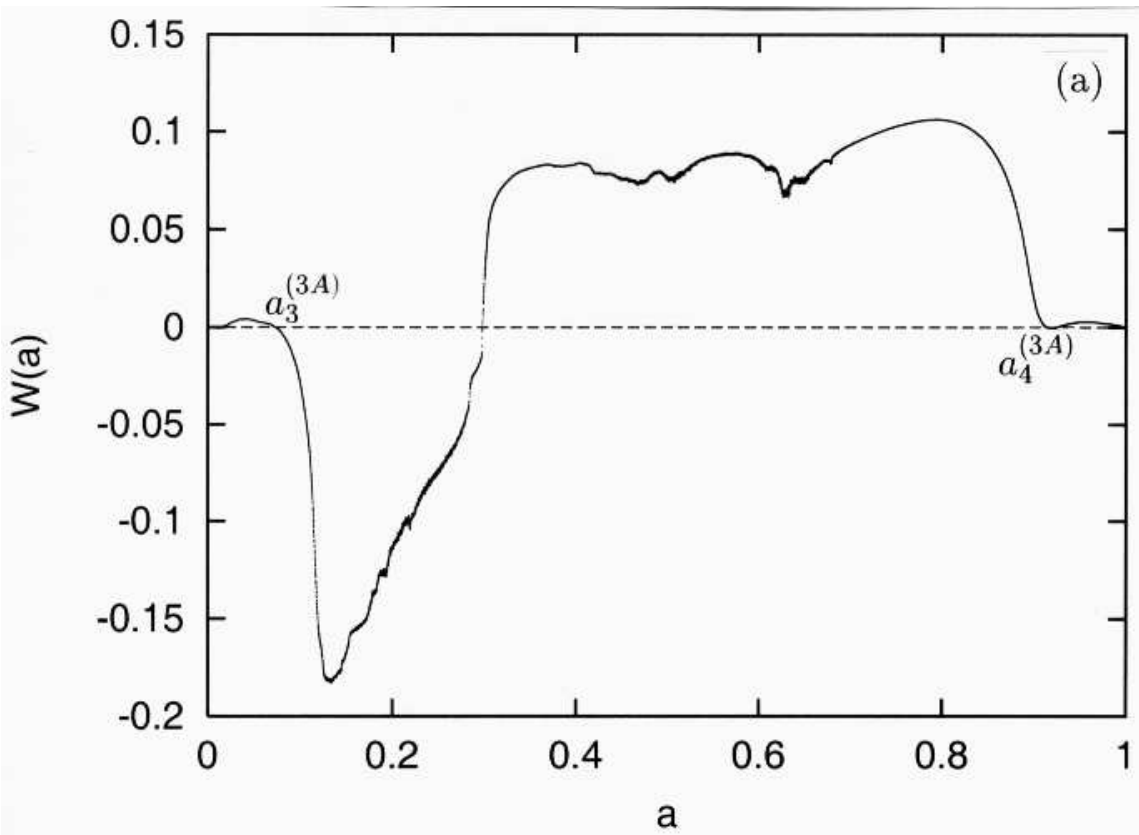

Fig. 3

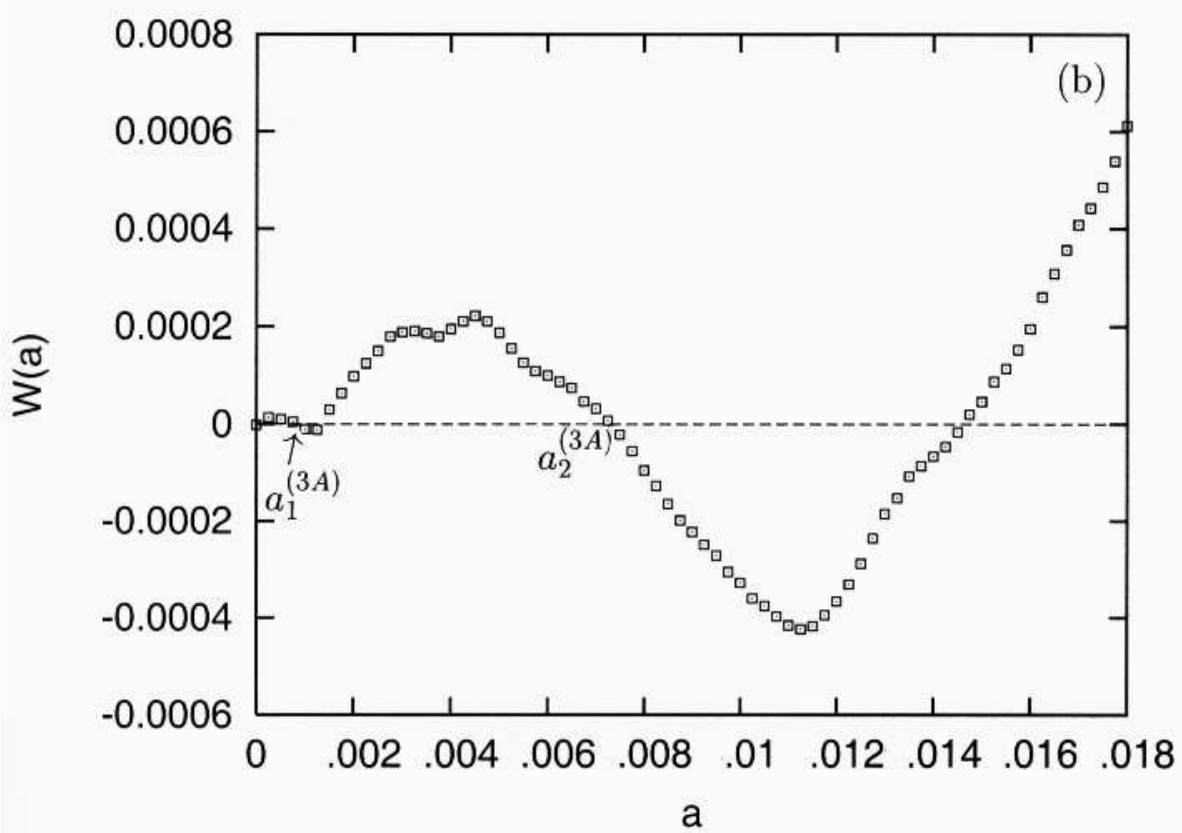




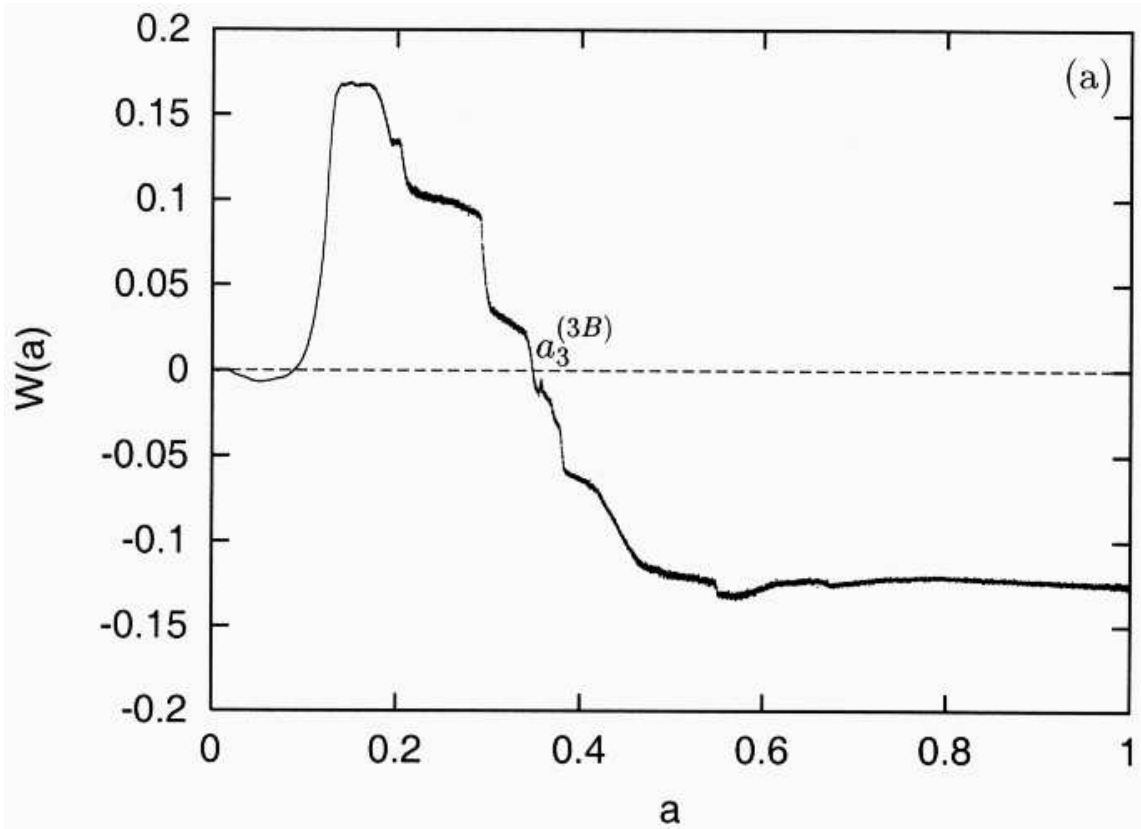

Fig. 4

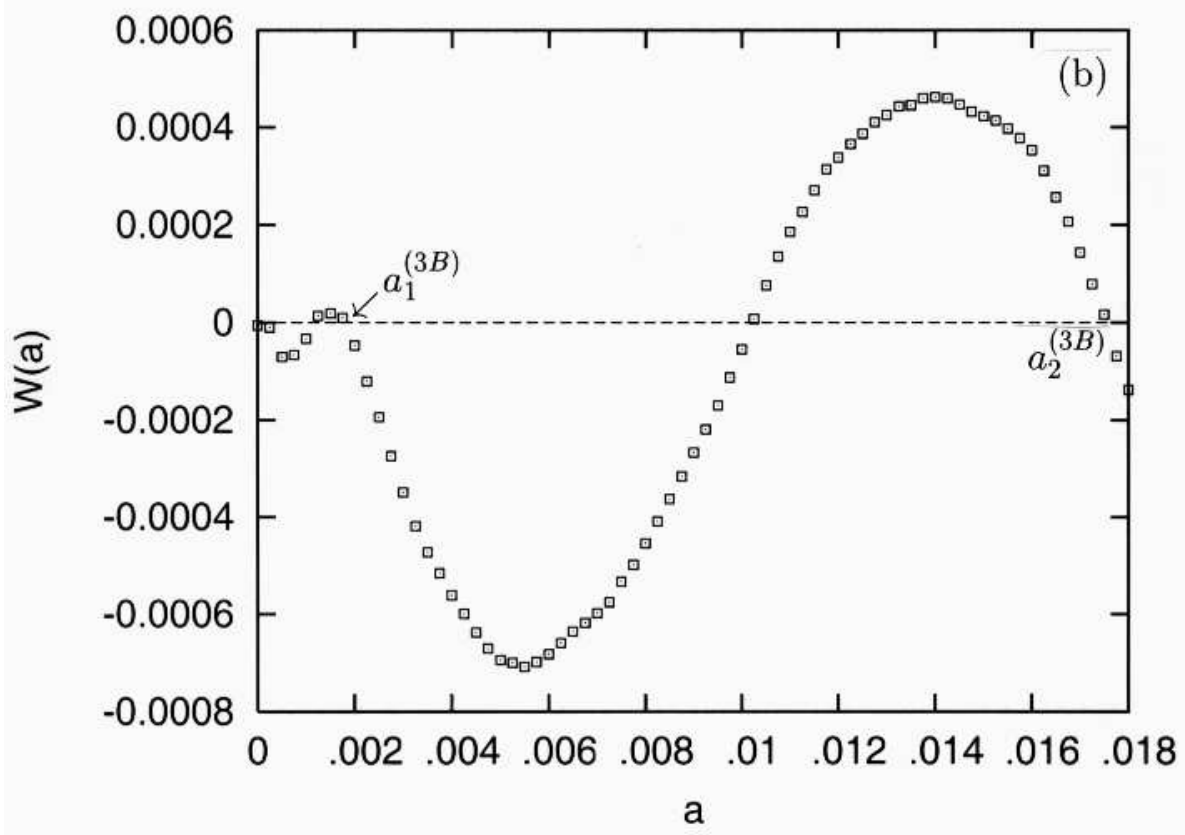




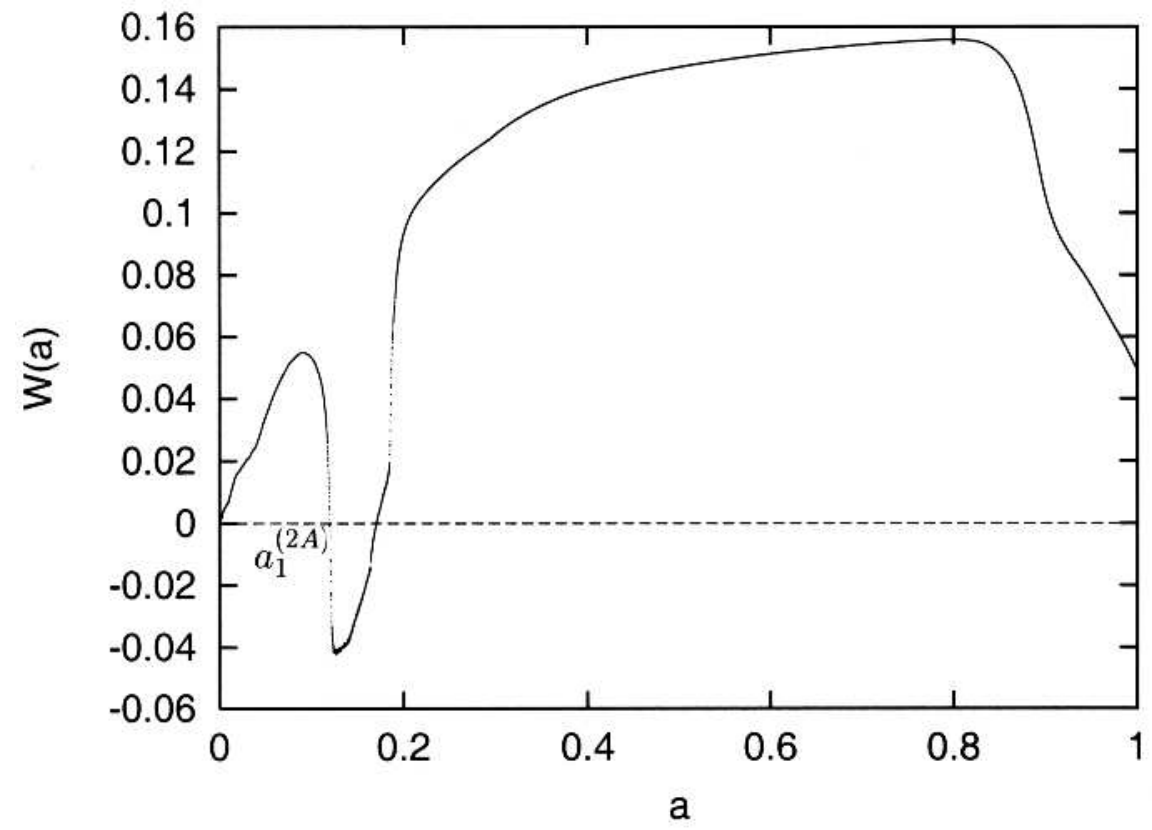

Fig. 5 


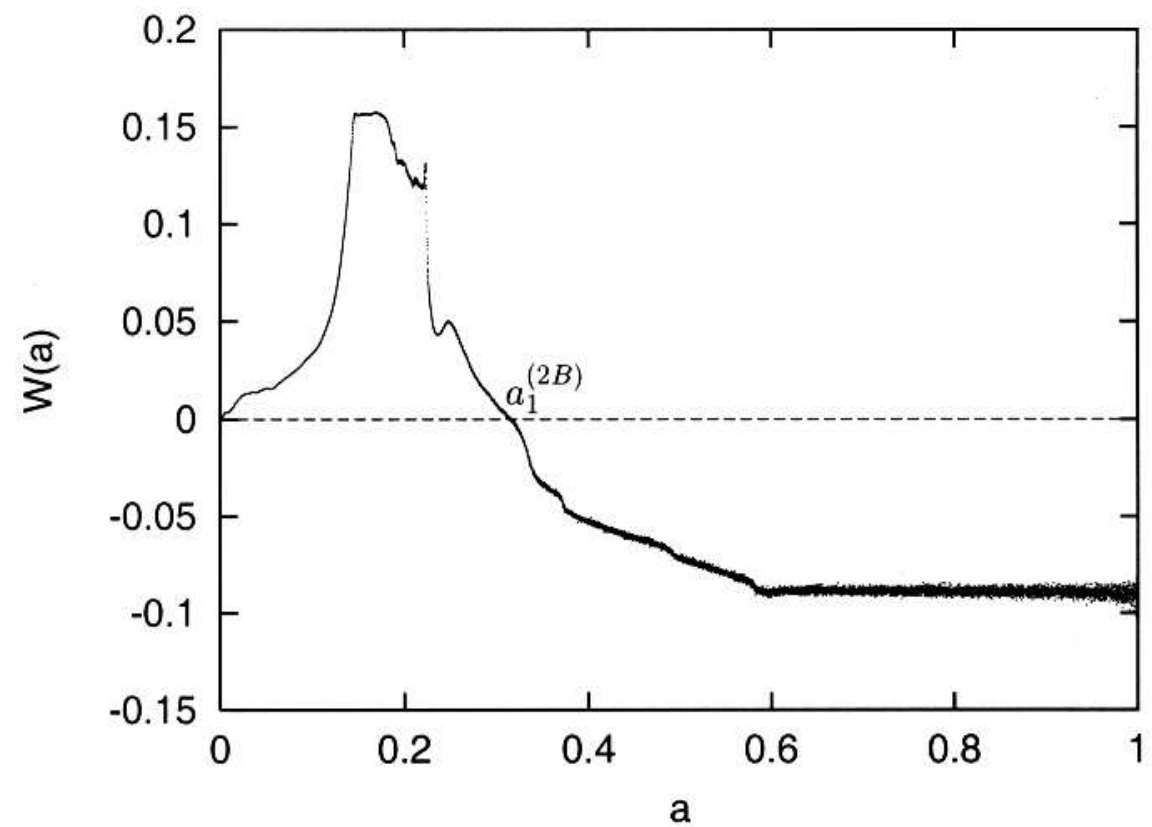

Fig. 6 


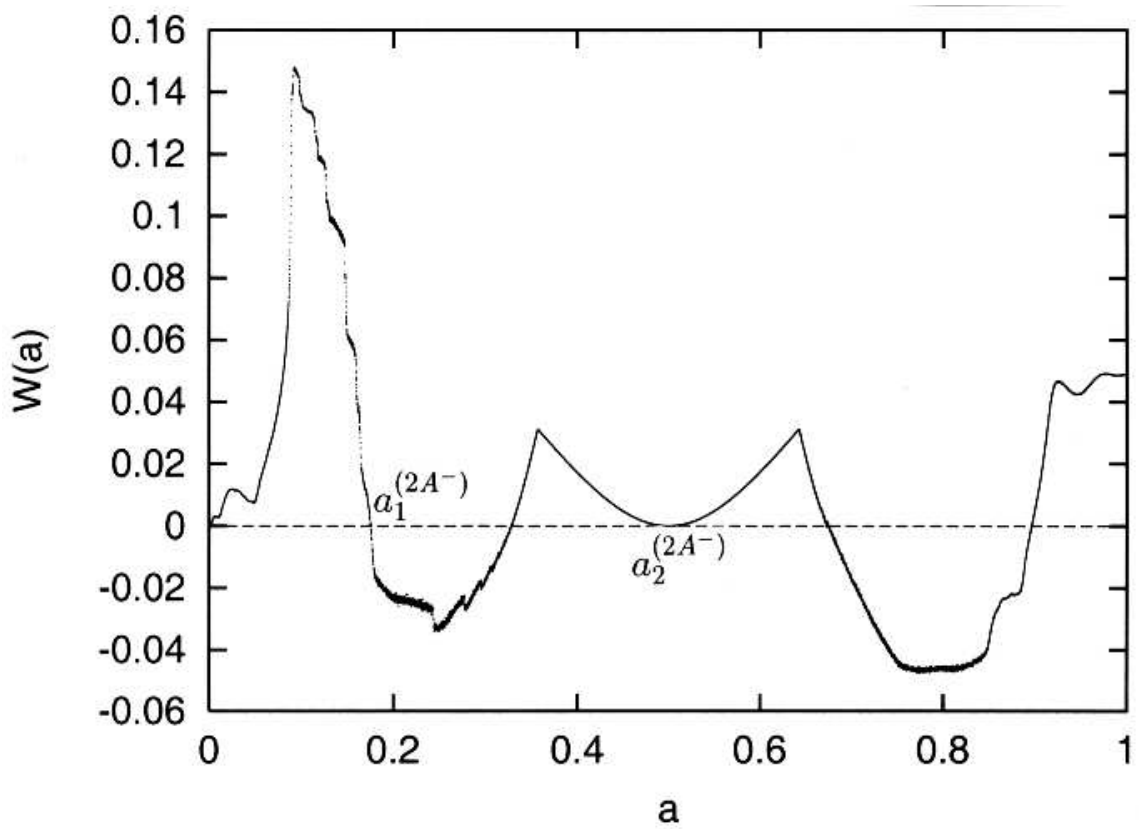

Fig. 7 


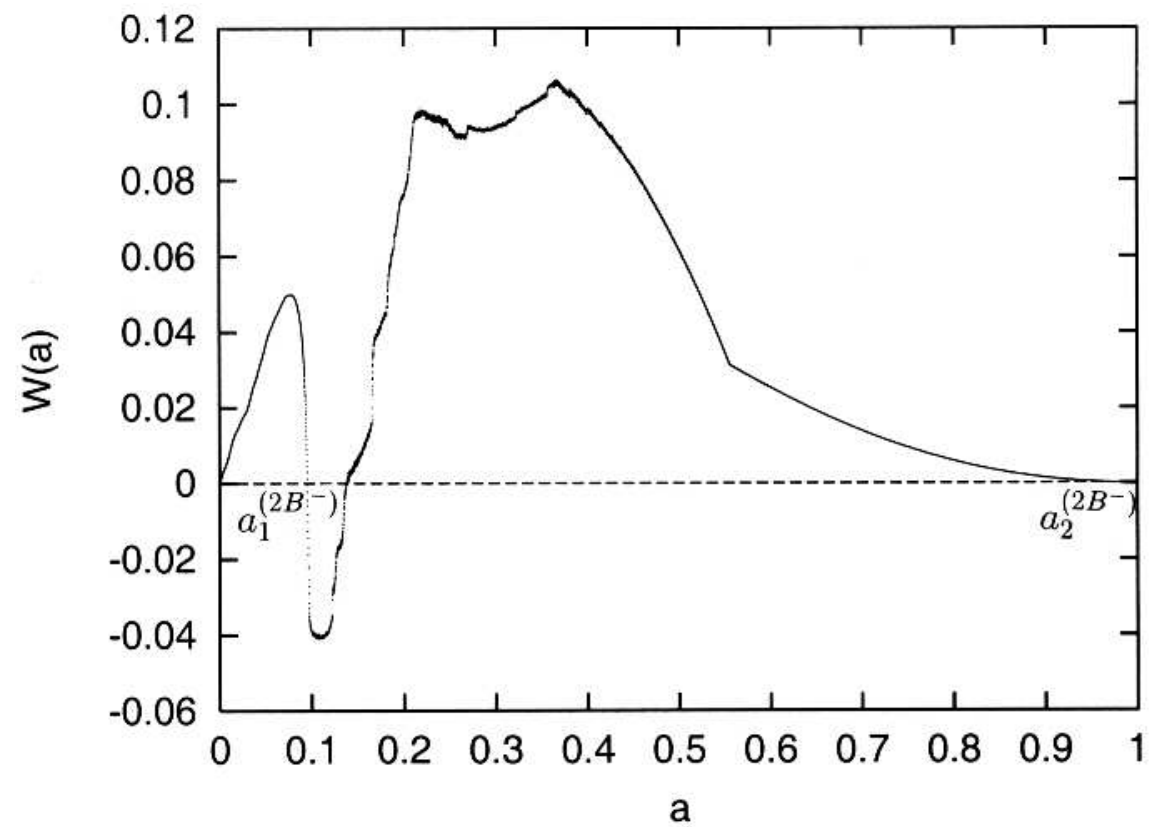

Fig. 8 


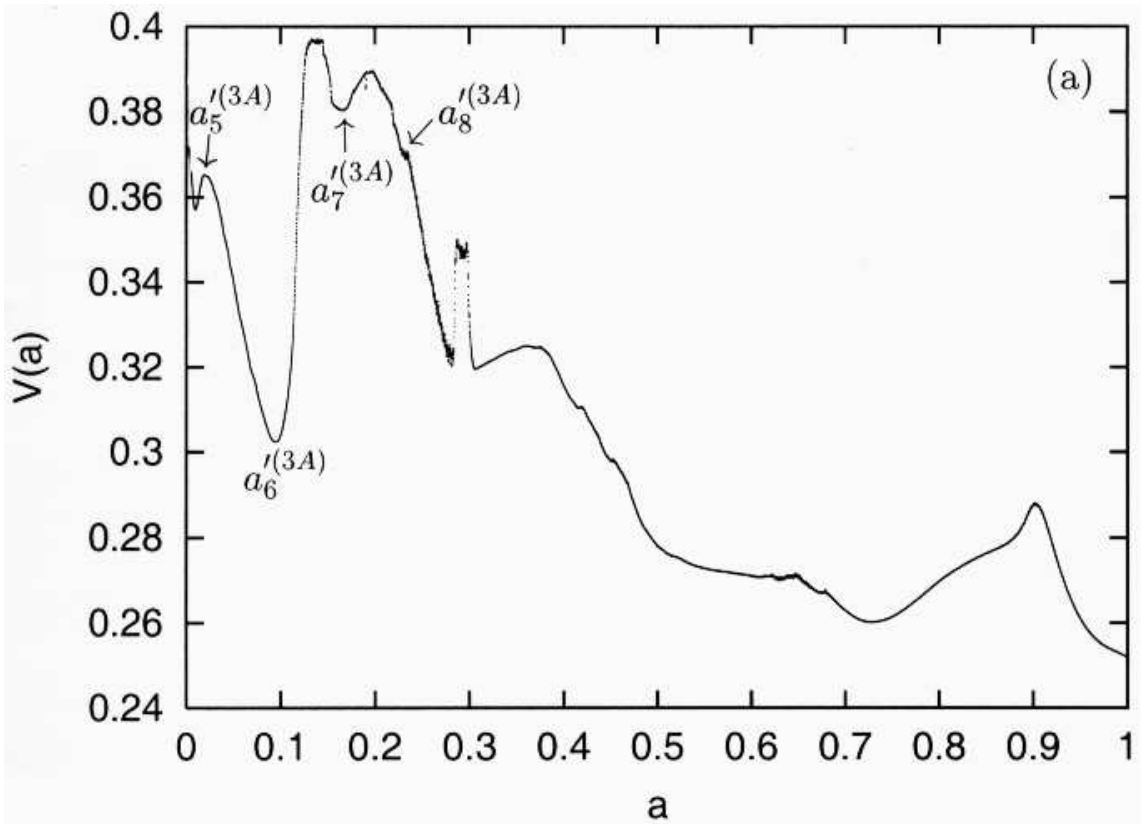

Fig. 9

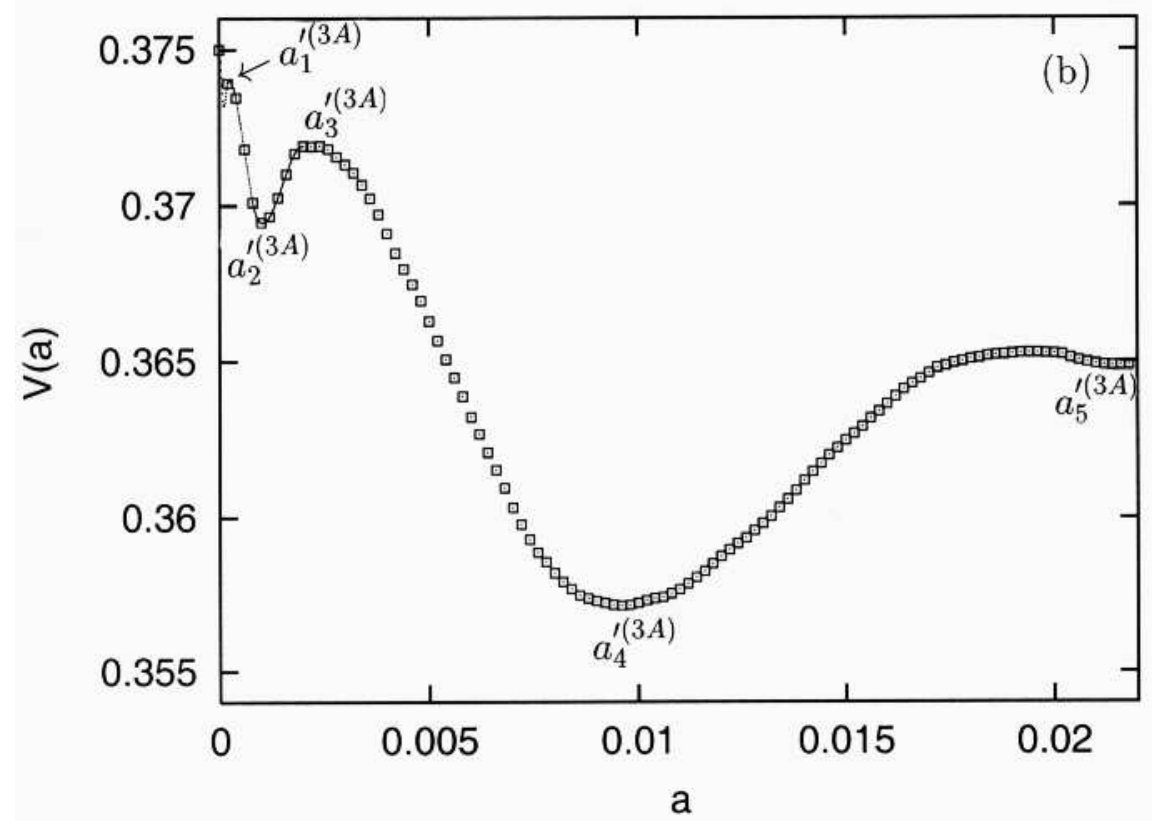




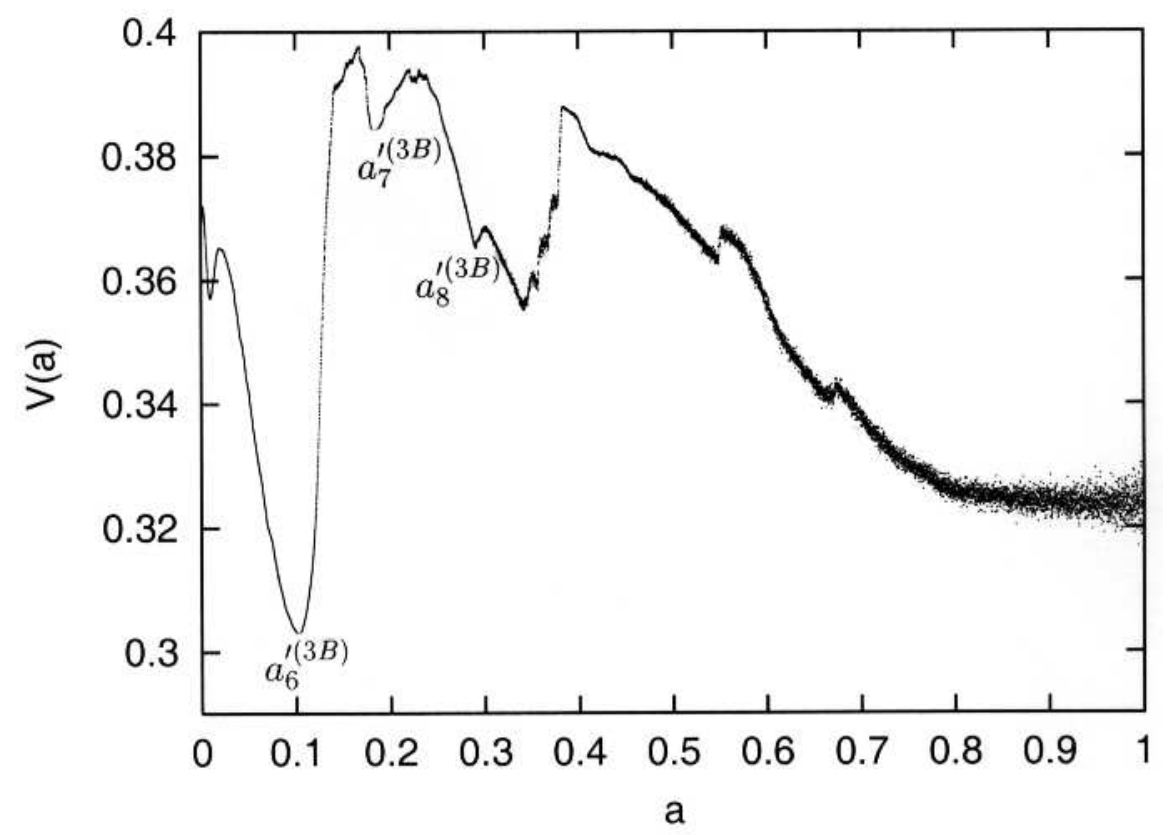

Fig. 10 


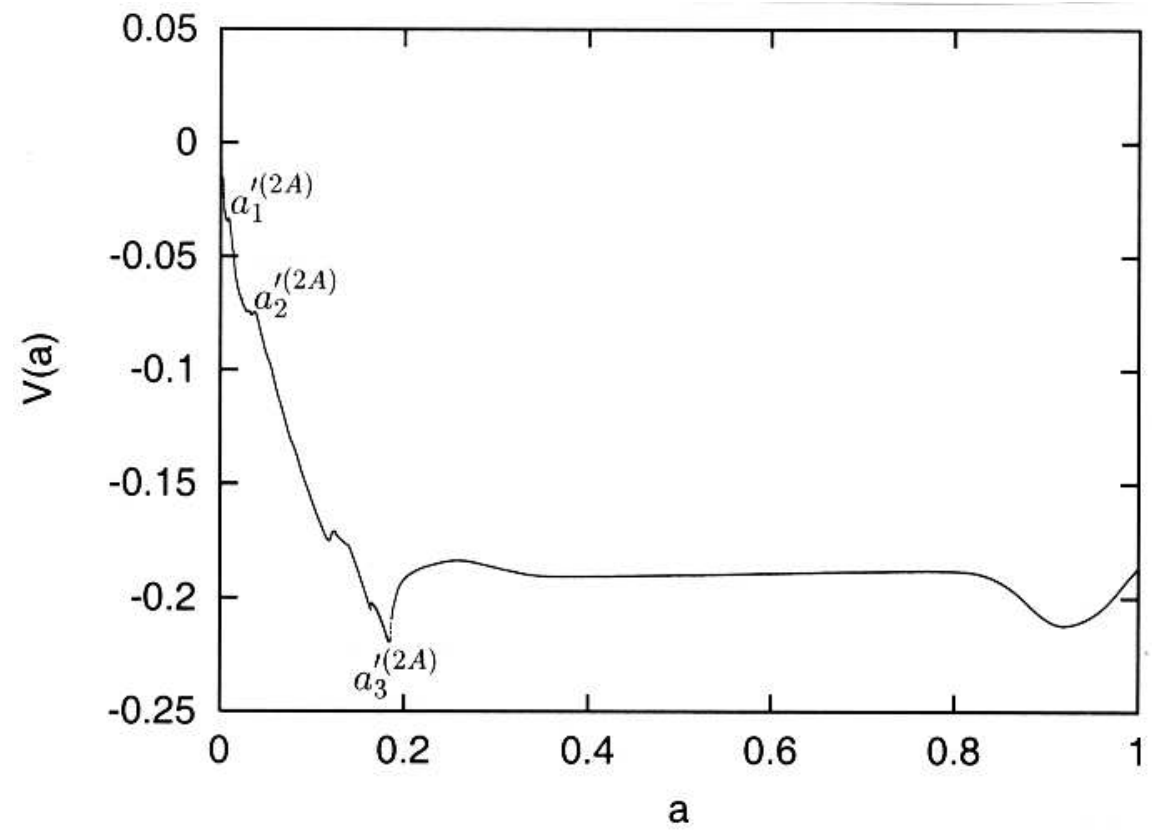

Fig. 11 


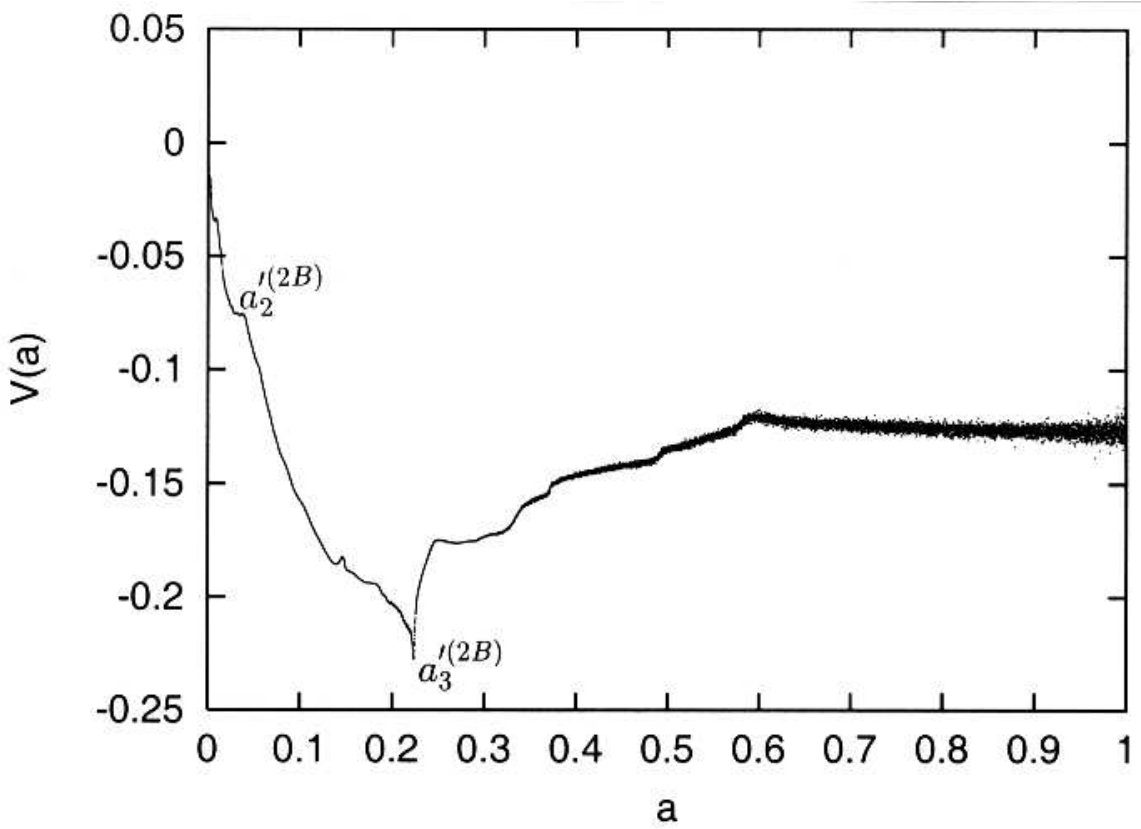

Fig. 12 


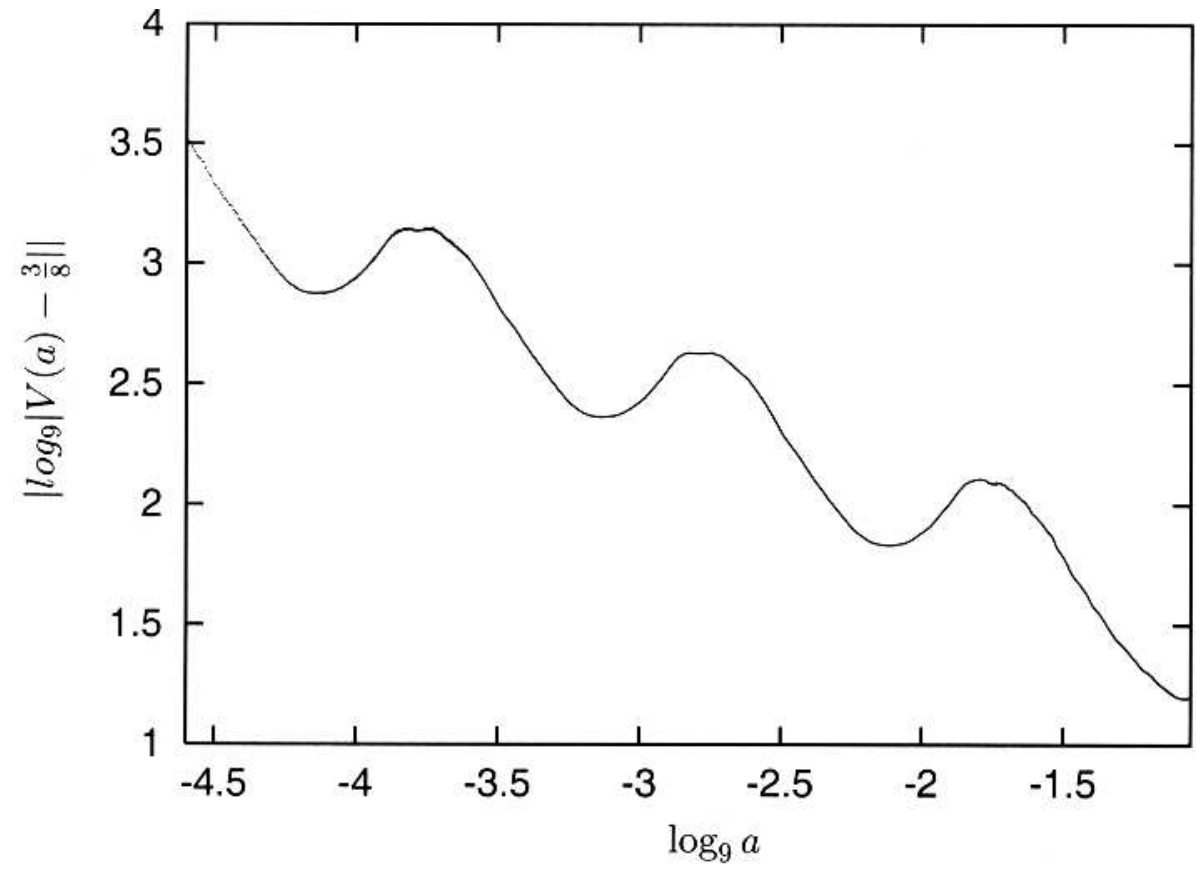

Fig. 13 


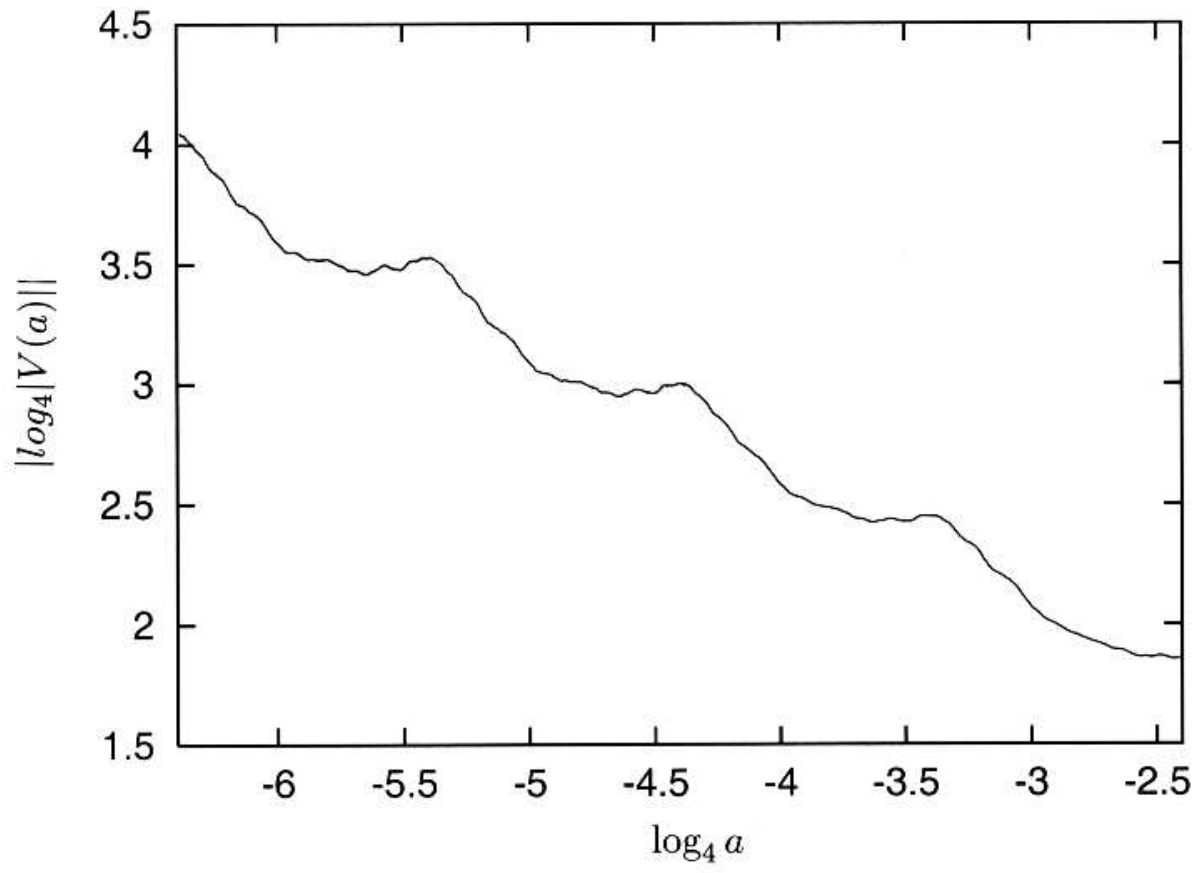

Fig. 14 


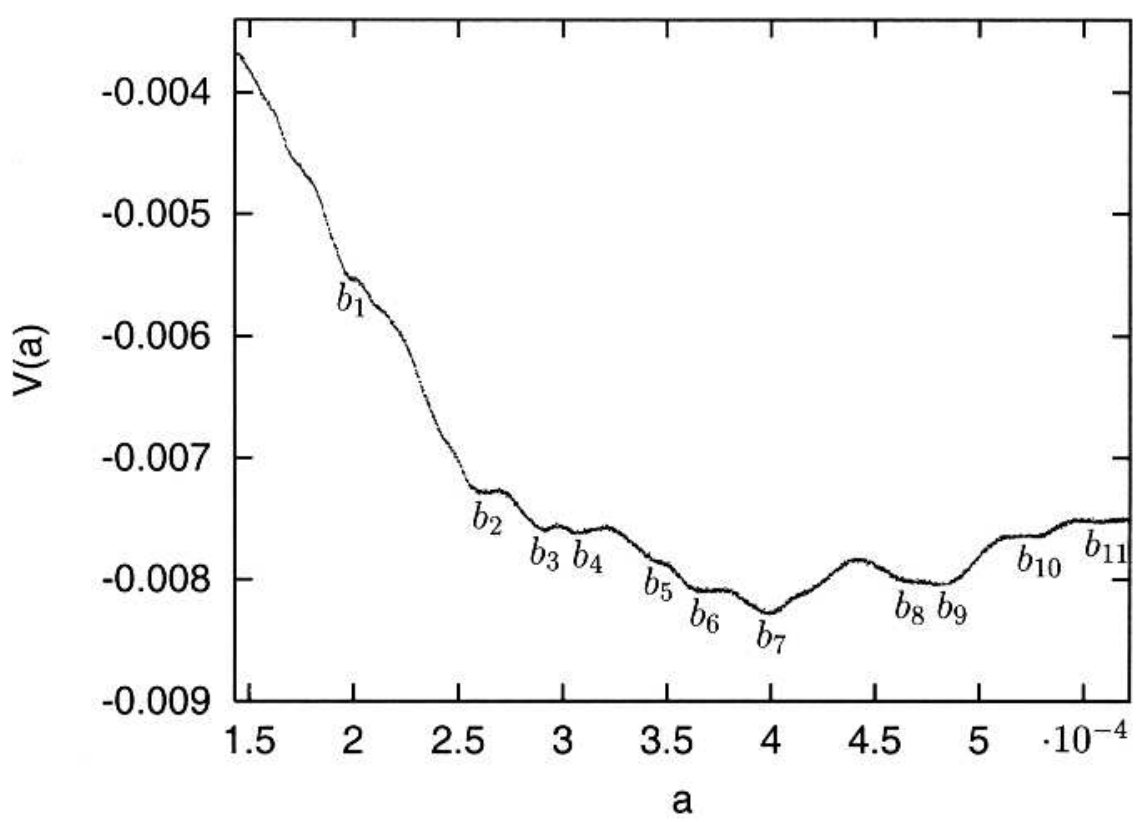

Fig. 15 


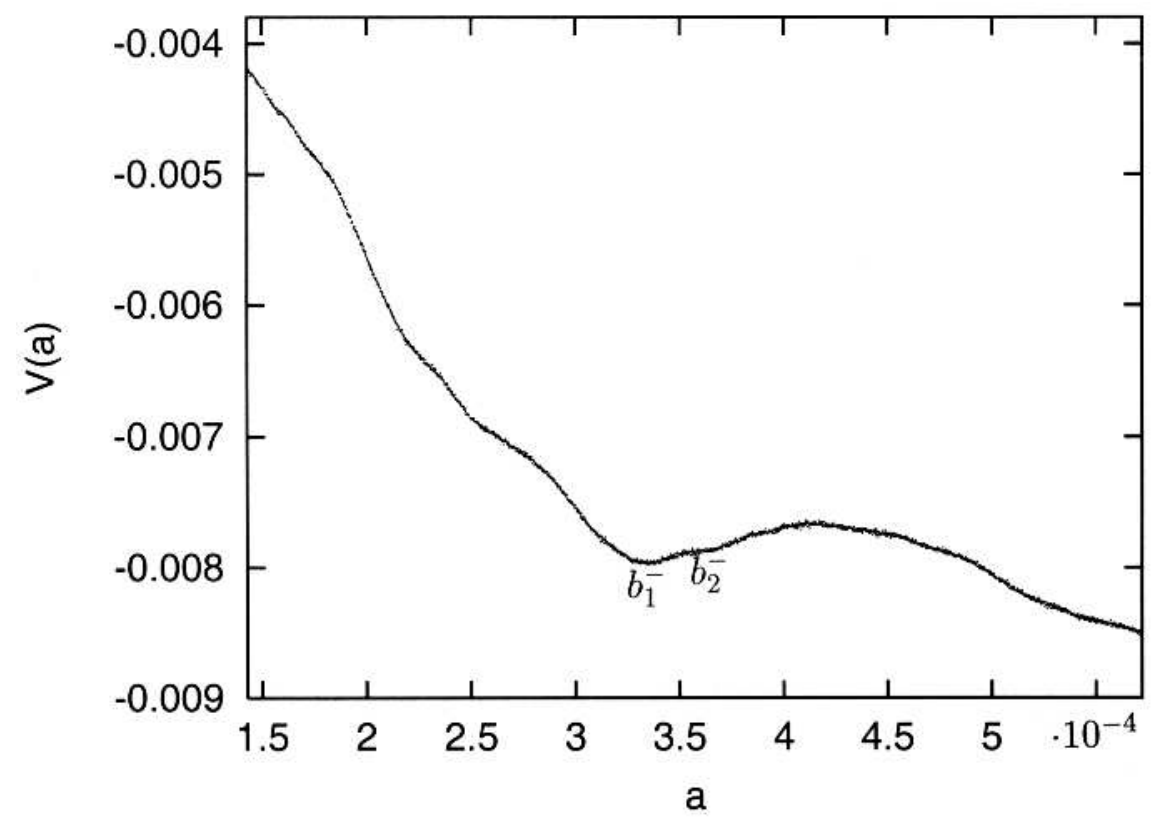

Fig. 16 
Interaction energy: coupling strengths of 4 interactions

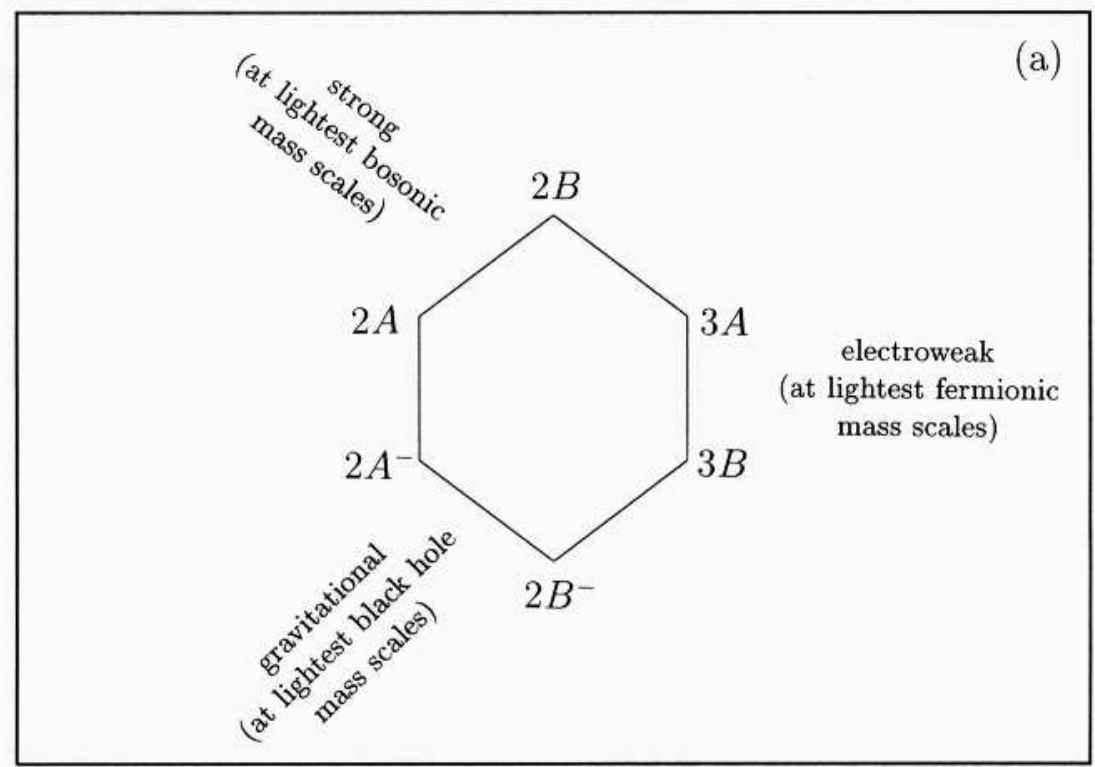

Fig. 17

Self energy: masses and mass mixing angles

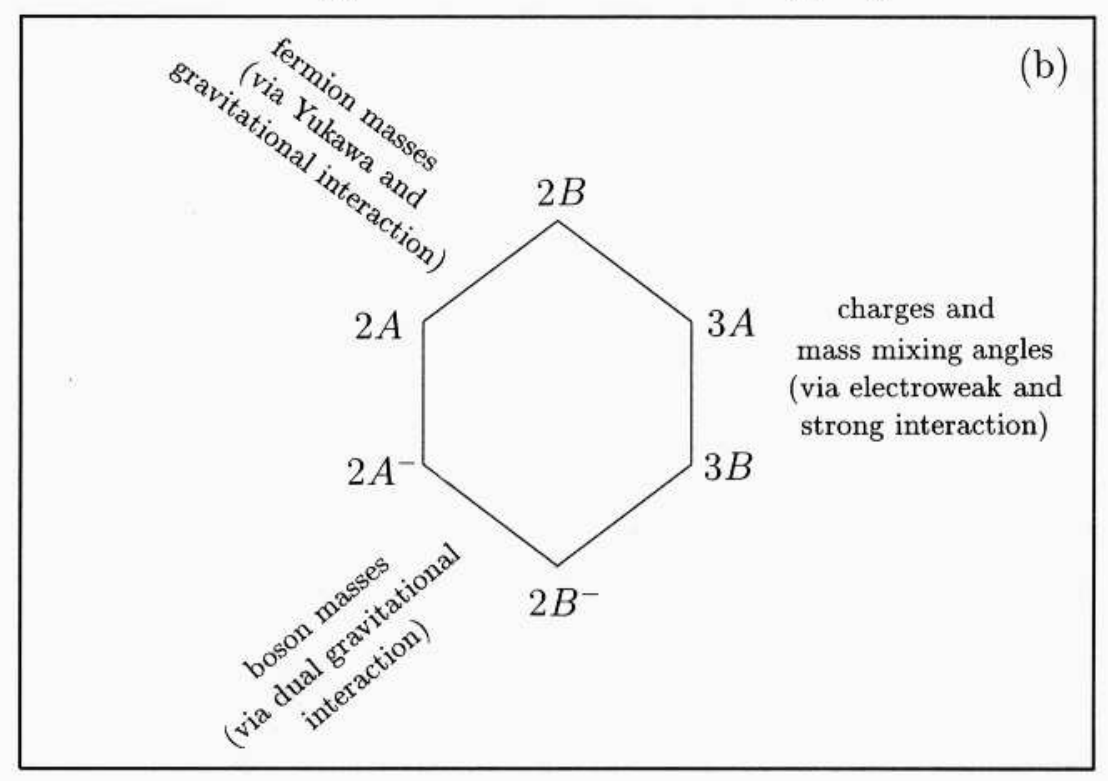




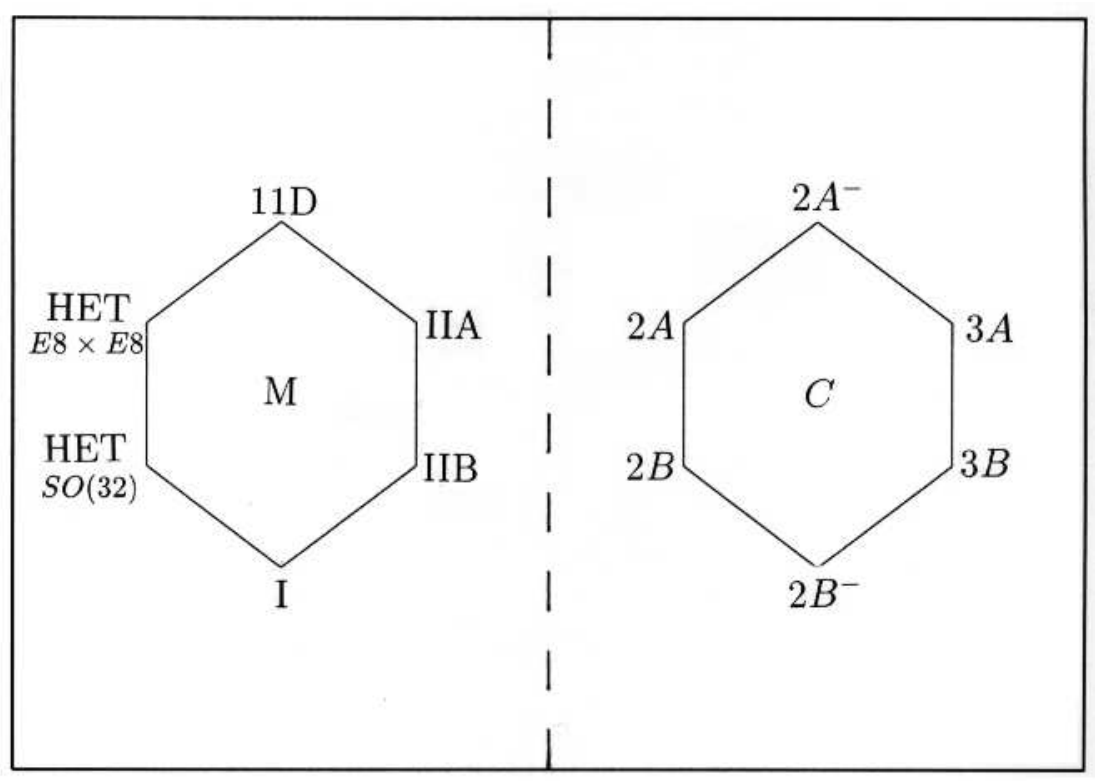

Fig. 18 